\title{
Local Growth Envelopes of Besov Spaces of Generalized Smoothness
}

\author{
Caetano, Antonio M ; Farkas, Walter
}

\begin{abstract}
The concept of local growth envelope (ELGA, u) of the quasi-normed function space A is applied to the Besov spaces of generalized smoothness B ,Np,q(Rn)
\end{abstract}

Posted at the Zurich Open Repository and Archive, University of Zurich ZORA URL: https://doi.org/10.5167/uzh-192353

Journal Article

Published Version

Originally published at:

Caetano, Antonio M; Farkas, Walter (2006). Local Growth Envelopes of Besov Spaces of Generalized Smoothness. Zeitschrift für Analysis und ihre Anwendungen, 25(3):265-298. 


\title{
Local Growth Envelopes of Besov Spaces of Generalized Smoothness
}

\author{
António M. Caetano and Walter Farkas
}

\begin{abstract}
The concept of local growth envelope $\left(\mathcal{E}_{\mathrm{LG}} A, u\right)$ of the quasi-normed function space $A$ is applied to the Besov spaces of generalized smoothness $B_{p, q}^{\sigma, N}\left(\mathbb{R}^{n}\right)$.

Keywords. Function space, generalized smoothness, atomic decomposition, growth envelope, sharp inequality, sharp embedding
\end{abstract}

Mathematics Subject Classification (2000). 46 E35

\section{Introduction}

The main aim of this paper is to give a unified approach to the question of determining the growth envelope for Besov spaces. It might be considered as an extension of [5], as far as Besov-type spaces are concerned. In fact, the techniques used to deal with the so-called critical case considered in that paperand quite different from the interpolation techniques used in [4] -, together with some breakthrough related to what should be the form of a local growth envelope function, opened the way to the consideration of the problem of determining the growth envelopes of function spaces of Besov type as general as the ones considered in [7].

Actually the idea of unification began in [5], where in some parts it was not necessary to distinguish between the so-called critical and subcritical cases; this is used in the present work, as for the general spaces we consider now it doesn't make much sense to make that distinction.

We are thus able to completely determine the growth envelopes of spaces of the type $B_{p, q}^{\sigma, N}\left(\mathbb{R}^{n}\right)$, apart from some limiting cases where the techniques break. However, the situation here is not much worse than what happens for the classical spaces $B_{p, q}^{s}\left(\mathbb{R}^{n}\right)$, as we also point out that, by specialising our

António M. Caetano: Departamento de Matemática, Universidade de Aveiro, 3810193 Aveiro, Portugal; e-mail: acaetano@mat.ua.pt

Walter Farkas: Swiss Banking Institute, University of Zurich, Plattenstr. 14, 8032

Zurich, Switzerland; e-mail: farkas@math.ethz.ch 
parameters in order to get the latter spaces, our limiting cases correspond to a limiting case for classical spaces where the picture is also not clear.

We give now a more detailed account of what is proved in the present paper. Functions spaces of generalized smoothness have been considered since the middle of the seventies of the last century, in particular by the Russian school, and have been again in the centre of interest in recent times. In particular because they are relevant in recent investigations in the theory of stochastic processes, where they appear in a natural way. For a short description of this and some historical remarks, we refer the interested reader to [7], where other relevant references can also be found.

The main objective here is to characterise the ability of local growth for functions of the spaces $B_{p, q}^{\sigma, N}\left(\mathbb{R}^{n}\right)$, when these spaces are not continuously embedded in $L_{\infty}$. This is partly done by studying the behaviour of the local growth envelope function

$$
\mathcal{E}_{\mathrm{LG}} \mid B_{p, q}^{\sigma, N}(t):=\sup \left\{f^{*}(t):\left\|f \mid B_{p, q}^{\sigma, N}\left(\mathbb{R}^{n}\right)\right\| \leqslant 1\right\}
$$

near 0 , where $f^{*}$ stands for the decreasing rearrangement of $f$ (so we need $f$ to be in $\left.L_{1}^{\text {loc }}\left(\mathbb{R}^{n}\right)\right)$.

When dealing with the classical Besov spaces $B_{p q}^{s}\left(\mathbb{R}^{n}\right)$ (which, in our general setting, correspond to $N=\left(2^{j}\right)_{j \in \mathbb{N}_{0}}$ and $\left.\sigma=\left(2^{j s}\right)_{j \in \mathbb{N}_{0}}\right)$, D. Haroske [19] and H. Triebel [33] have proved that $\mathcal{E}_{\mathrm{LG}} \mid B_{p q}^{s}(t)$ behaves like $t^{\frac{s}{n}-\frac{1}{p}}$ near 0 in the subcritical case and like $|\log t|^{\frac{1}{q^{\prime}}}$ near 0 in the critical case (with $q^{\prime}$ standing for the conjugate exponent of $q$ and where $q$ is here assumed to be greater than 1 , as otherwise the question is of no interest).

In [4] and [5], A. Caetano and S. Moura proved that if $N=\left(2^{j}\right)_{j \in \mathbb{N}_{0}}$ and $\sigma=\left(2^{j s} \Psi\left(2^{-j}\right)\right)_{s \in \mathbb{N}_{0}}$, for a so-called admissible function $\Psi$ in the context of those papers, the corresponding $\mathcal{E}_{\mathrm{LG}} \mid B_{p, q}^{\sigma, N}(t)$ behaves like

$$
\left(\int_{t^{\frac{1}{n}}}^{1} y^{\left(s-\frac{n}{p}\right) q^{\prime}} \Psi(y)^{-q^{\prime}} \frac{d y}{y}\right)^{\frac{1}{q^{\prime}}}
$$

near 0 , with some natural restrictions and conventions. They also prove there that this seemingly complicated expression reduces to simple ones in some important special cases, so that it is possible to recover the expressions obtained previously by D. Haroske and H. Triebel in the classical context. However, the expression (1.1) is of interest in our broader context, because of its ability to be generalized. In fact, with natural restrictions and off 'limiting' cases (see Section 4.4 for the precise assertion and explanations), we show here that the behaviour of general $\mathcal{E}_{\mathrm{LG}} \mid B_{p, q}^{\sigma, N}(t)$ near 0 is

$$
\left(\int_{t^{\frac{1}{n}}}^{1} y^{-\frac{n}{p} q^{\prime}} \Lambda\left(y^{-1}\right)^{-q^{\prime}} \frac{d y}{y}\right)^{\frac{1}{q^{\prime}}},
$$


for some suitable function $\Lambda$ which, in particular, satisfies the relation $\Lambda\left(N_{j}\right) \sim$ $\sigma_{j}, j \in \mathbb{N}_{0}$.

Following the ideas of D. Haroske from [19] and of H. Triebel from [33] when introducing the concept of local growth envelope, and denoting by $\Phi_{q^{\prime}}(t)$ the expression (1.2), we also study the behaviour of an individual $f^{*}$ against $\Phi_{q^{\prime}}$ and the Borel measure $\mu_{q^{\prime}}$ associated with $-\log _{2} \Phi_{q^{\prime}}$ in some interval $(0, \varepsilon]$ (for some small positive $\varepsilon$ ), thus proving that the best exponent $v$ such that

$$
\left(\int_{0}^{\varepsilon}\left(\frac{f^{*}(t)}{\Phi_{q^{\prime}}(t)}\right)^{v} \mu_{q^{\prime}}(d t)\right)^{\frac{1}{v}} \leqslant c\left\|f \mid B_{p, q}^{\sigma, N}\left(\mathbb{R}^{n}\right)\right\|,
$$

for some constant $c=c(v)$ and all $f \in B_{p, q}^{\sigma, N}$, is $q$, just like in the classical setting. Notice that, when $N=\left(2^{j}\right)_{j \in \mathbb{N}_{0}}, \sigma=\left(2^{j n / p}\right)_{j \in \mathbb{N}_{0}}$ and $v=q$, (1.3) can be written as

$$
\left(\int_{0}^{\varepsilon}\left(\frac{f^{*}(t)}{\left|\log _{2} t\right|}\right)^{q} \frac{d t}{t}\right)^{\frac{1}{q}} \leqslant c\left\|f \mid B_{p, q}^{n / p}\left(\mathbb{R}^{n}\right)\right\|,
$$

which may become apparent that we are pushing forward in a direction already followed by many other authors. We refer the reader to [33, $11.8(\mathrm{v}), 13.5]$ for historical references to the subject and related more recent developments, where the names of Adams, Brézis, Brudnyi, Cwikel, Edmunds, Gold'man, Hansson, Kaljabin, Kerman, Krbec, Maz'ya, Moser, Netrusov, Peetre, Pick, Pohozaev, Pustylnik, Schmeisser, Strichartz, Triebel, Trudinger, Wainger, Yudovich and Ziemer are cited. See also related recent results of B. Opic and W. Trebels [27] and P. Gurka and B. Opic [17].

\section{Preliminaries}

\subsection{Sequences.}

Assumption 1. By an admissible sequence we will always mean a sequence $\gamma=\left(\gamma_{j}\right)_{j \in \mathbb{N}_{0}}$ of positive numbers such that there are two constants $0<\kappa_{0} \leqslant$ $\kappa_{1}<\infty$ with

$$
\kappa_{0} \gamma_{j} \leqslant \gamma_{j+1} \leqslant \kappa_{1} \gamma_{j} \text { for any } j \in \mathbb{N}_{0} .
$$

We shall need the following notation with respect to an admissible sequence

$$
\underline{\gamma}_{j}:=\inf _{k \geqslant 0} \frac{\gamma_{j+k}}{\gamma_{k}} \quad \text { and } \quad \bar{\gamma}_{j}:=\sup _{k \geqslant 0} \frac{\gamma_{j+k}}{\gamma_{k}}, \quad j \in \mathbb{N}_{0} .
$$

Note that, in particular, $\underline{\gamma}_{1}$ and $\bar{\gamma}_{1}$ are the best constants $\kappa_{0}$ and $\kappa_{1}$ in $(2.1)$, respectively. 
To illustrate the flexibility of (2.1) we recall below an example discussed in $[7]$.

Example 2.1. Let $s \in \mathbb{R}$ be fixed and

$$
\gamma_{j}=2^{j s} \Psi\left(2^{-j}\right), \quad j \in \mathbb{N}_{0},
$$

where $\Psi$ is a positive monotone function on $(0,1]$, and there are positive constants $b_{0}$ and $b_{1}$ such that for all $j \in \mathbb{N}_{0}$

$$
b_{0} \Psi\left(2^{-j}\right) \leqslant \Psi\left(2^{-2 j}\right) \leqslant b_{1} \Psi\left(2^{-j}\right) .
$$

Then it is easy to see that $\gamma$ is an admissible sequence: we can take $\kappa_{0}=$ $\min \left(b_{0}, 1, \Psi\left(2^{-1}\right) \Psi(1)^{-1}\right) \cdot 2^{s}$ and $\kappa_{1}=\max \left(b_{1}, 1, \Psi\left(2^{-1}\right) \Psi(1)^{-1}\right) \cdot 2^{s}$ in $(2.1)$.

2.2. Functions. The functions we are going to introduce now will be central in the estimates which will be presented later.

Definition 2.2. A function $\Lambda:(0, \infty) \rightarrow(0, \infty)$ will be called admissible if it is continuous and if for any $b>0$ it satisfies

$$
\Lambda(b z) \sim \Lambda(z) \text { for any } z>0,
$$

in the sense that for any $b>0$ there exist $c_{1}, c_{2}>0$ such that $c_{1} \Lambda(z) \leqslant \Lambda(b z) \leqslant$ $c_{2} \Lambda(z)$ for any $z>0$.

Example 2.3. Let $\left(N_{j}\right)_{j \in \mathbb{N}_{0}}$ be a sequence of positive numbers such that for some $\lambda_{0}>1$ it holds $\lambda_{0} N_{j} \leqslant N_{j+1}$ for all $j \in \mathbb{N}_{0}$. Let $\left(\sigma_{j}\right)_{j \in \mathbb{N}_{0}}$ be an admissible sequence. Then the function $\Lambda:(0, \infty) \rightarrow(0, \infty)$ defined by

$$
\Lambda(z)= \begin{cases}\frac{\sigma_{j+1}-\sigma_{j}}{N_{j+1}-N_{j}} z+\sigma_{j}-\frac{\left(\sigma_{j+1}-\sigma_{j}\right) N_{j}}{N_{j+1}-N_{j}}, & \text { if } z \in\left[N_{j}, N_{j+1}\right), j \in \mathbb{N}_{0} \\ \sigma_{0}, & \text { if } z \in\left(0, N_{0}\right)\end{cases}
$$

is admissible and satisfies $\Lambda\left(N_{j}\right)=\sigma_{j}$ for any $j \in \mathbb{N}_{0}$. Moreover, $\Lambda(z) \sim \sigma_{j}$ for $z \in\left[N_{j}, N_{j+1}\right], j \in \mathbb{N}_{0}$, with equivalence constants independent of $j$.

Definition 2.4. Let $n \in \mathbb{N}$ and $0<p \leqslant \infty$ have been fixed. Let $\Lambda:(0, \infty) \rightarrow$ $(0, \infty)$ be an admissible function and $0<\omega<1$. For $u \in(0, \infty]$ we define $\Phi_{u}:(0,1-\omega] \rightarrow \mathbb{R}$ by

$$
\Phi_{u}(t):=\left(\int_{t^{\frac{1}{n}}}^{1} y^{-\frac{n}{p} u} \Lambda\left(y^{-1}\right)^{-u} \frac{d y}{y}\right)^{\frac{1}{u}}, \quad \text { if } 0<u<\infty
$$

and

$$
\Phi_{u}(t):=\sup _{t^{\frac{1}{n}} \leqslant y \leqslant 1} y^{-\frac{n}{p}} \Lambda\left(y^{-1}\right)^{-1}, \quad \text { if } u=\infty .
$$


Lemma 2.5. The function $\Phi_{u}$ from Definition 2.4 is positive, monotonically decreasing and continuous. If $u \neq \infty$ then $\Phi_{u}$ is differentiable and its derivative is given by

$$
\Phi_{u}^{\prime}(t)=-\frac{1}{u n} t^{-\frac{u}{p}-1} \Lambda\left(t^{-\frac{1}{n}}\right)^{-u} \Phi_{u}(t)^{1-u}, \quad t \in(0,1-\omega] .
$$

We omit the proof of this lemma, as it is quite similar to the proof of Proposition 2.5 in [5].

Lemma 2.6. Let $\Lambda:(0, \infty) \rightarrow(0, \infty)$ be an admissible function and $0<\omega<1$, and let $\Phi_{u}$ be the function from Definition 2.4. Then for any constants $C>0$ and $t_{0}:=\frac{1-\omega}{\max \{1, C\}}$ the equivalence $\Phi_{u}(C t) \sim \Phi_{u}(t)$ holds for $t \in\left(0, t_{0}\right]$ in the sense that there exist two constants $c_{1}, c_{2}>0$ such that $c_{1} \Phi_{u}(t) \leqslant \Phi_{u}(C t) \leqslant$ $c_{2} \Phi_{u}(t)$ for any such $t$.

The proof of this lemma is based essentially on property (2.3) and is quite elementary. The same can be said about the following proposition, the proof of which can, moreover, be adapted from a corresponding discretization result in [5, Proposition 2.7]. Both results will be quite useful in the sequel.

Proposition 2.7. Let $\left(N_{j}\right)_{j \in \mathbb{N}_{0}}$ be as in Assumption 2 below. Let $\Lambda:(0, \infty) \rightarrow$ $(0, \infty)$ be an admissible function such that $\Lambda(z) \sim \Lambda\left(N_{j}\right), z \in\left[N_{j}, N_{j+1}\right], j \in$ $\mathbb{N}_{0}$, with equivalence constants independent of $j$. Let $\Phi_{u}$ be the function from Definition 2.4 with $\omega=1-N_{J_{0}}^{-n}$, where $J_{0} \in \mathbb{N}_{0}$ satisfies $N_{J_{0}}>1$. Given $t \in\left(0, N_{J_{0}}^{-n}\right]$, let $k=k(t)$ be the unique nonnegative integer such that $N_{k+1}^{-1}<$ $t^{\frac{1}{n}} \leqslant N_{k}^{-1}$. Let $0<u<\infty$. Given any integer $j_{0} \geqslant J_{0}$,

$$
\Phi_{u}(t) \sim\left(\sum_{j=j_{0}}^{k}\left(N_{j}^{\frac{n}{p}} \Lambda\left(N_{j}\right)^{-1}\right)^{u}\right)^{\frac{1}{u}} \quad \text { in }\left(0, N_{j_{0}}^{-n}\right] .
$$

In particular, for any $k_{0} \in \mathbb{N}$ and any $K_{0} \in \mathbb{N}$ we have

$$
\Phi_{u}(t) \sim\left(\sum_{j=j_{0}}^{k-k_{0}}\left(N_{j}^{\frac{n}{p}} \Lambda\left(N_{j}\right)^{-1}\right)^{u}\right)^{\frac{1}{u}} \quad \text { in }\left(0, N_{j_{0}+k_{0}}^{-n}\right]
$$

and

$$
\Phi_{u}(t) \sim\left(\sum_{j=j_{0}}^{k+K_{0}}\left(N_{j}^{\frac{n}{p}} \Lambda\left(N_{j}\right)^{-1}\right)^{u}\right)^{\frac{1}{u}} \quad \text { in }\left(0, N_{j_{0}^{\prime}}^{-n}\right]
$$

where $j_{0}^{\prime}:=\max \left(j_{0}-K_{0}, J_{0}\right)$. If $u=\infty$, then the right-hand sides of the equivalences stated above are replaced by $\sup _{j}\left(N_{j}^{\frac{n}{p}} \Lambda\left(N_{j}\right)^{-1}\right)$, the supremum being taken over corresponding j's. 
Remark 2.8. It might be useful to note that the summations stated in the proposition above can also start in 0 or in any number between 0 and $j_{0}$. The situation is similar in the integral representation for $\Phi_{u}$ in Definition 2.4: we can as well choose for upper limit of integration any real number greater than 1 , in the sense of equivalent representations.

\section{Function spaces of generalized smoothness}

Function spaces of generalized smoothness have been introduced and considered by several authors, in particular since the middle of the seventies up to the end of the eighties with different starting points and in different contexts.

In [7] some of these different aspects concerning function spaces of generalized smoothness were discussed taking up some basic ideas from various settings but from the standpoint of a Fourier analytic characterisation. In particular in [7] an atomic decomposition for spaces of generalized smoothness was obtained and that result will play a key role in our later considerations, see Theorem 3.14 below.

\subsection{Definitions and basic facts.}

Assumption 2. From now on we will denote $N=\left(N_{j}\right)_{j \in \mathbb{N}_{0}}$ a sequence of real positive numbers such that there exist two numbers $1<\lambda_{0} \leqslant \lambda_{1}$ with

$$
\lambda_{0} N_{j} \leqslant N_{j+1} \leqslant \lambda_{1} N_{j} \text { for any } j \in \mathbb{N}_{0} .
$$

In particular $N$ is admissible and is a so-called strongly increasing sequence (compare [7, Definition 2.2.1]), which in particular guarantees that there exists a number $l_{0} \in \mathbb{N}$ such that

$$
2 N_{j} \leqslant N_{k} \text { for any } l, k \text { such that } j+l_{0} \leqslant k .
$$

We would like to point out that the condition $\lambda_{0}>1$ played a key role in [7] in order to get atomic decompositions in function spaces of generalized smoothness.

It should be noted that the sequence $N=\left(N_{j}\right)_{j \in \mathbb{N}_{0}}$ plays the same role as the sequence $\left(2^{j}\right)_{j \in \mathbb{N}_{0}}$ in the classical construction of the spaces $B_{p, q}^{s}$ and $F_{p, q}^{s}$. This will be clear from the following considerations.

For a fixed sequence $N=\left(N_{j}\right)_{j \in \mathbb{N}_{0}}$ as in Assumption 2 we define the associated covering $\Omega^{N}=\left(\Omega_{j}^{N}\right)_{j \in \mathbb{N}_{0}}$ of $\mathbb{R}^{n}$ by

$$
\Omega_{j}^{N}=\left\{\xi \in \mathbb{R}^{n}:|\xi| \leqslant N_{j+l_{0}}\right\}, \quad \text { if } j=0,1, \cdots l_{0}-1
$$

and

$$
\Omega_{j}^{N}=\left\{\xi \in \mathbb{R}^{n}: N_{j-l_{0}} \leqslant|\xi| \leqslant N_{j+l_{0}}\right\}, \quad \text { if } j \geqslant l_{0}
$$

where $l_{0}$ was defined in $(3.2)$. 
Definition 3.1. For a fixed $N=\left(N_{j}\right)_{j \in \mathbb{N}_{0}}$ as in Assumption 2, and for the associated covering $\Omega^{N}=\left(\Omega_{j}^{N}\right)_{j \in \mathbb{N}_{0}}$ of $\mathbb{R}^{n}$, a system $\varphi^{N}=\left(\varphi_{j}^{N}\right)_{j \in \mathbb{N}_{0}}$ will be called a (generalized) partition of unity subordinated to $\Omega^{N}$ if:

(i) for any $j \in \mathbb{N}_{0}$,

$$
\varphi_{j}^{N} \in C_{0}^{\infty}\left(\mathbb{R}^{n}\right) \quad \text { and } \quad \varphi_{j}^{N}(\xi) \geqslant 0 \quad \text { if } \xi \in \mathbb{R}^{n}
$$

(ii) for any $j \in \mathbb{N}_{0}$,

$$
\operatorname{supp} \varphi_{j}^{N} \subset \Omega_{j}^{N}
$$

(iii) for any $\gamma \in \mathbb{N}_{0}^{n}$ there exists a constant $c_{\gamma}>0$ such that for any $j \in \mathbb{N}_{0}$,

$$
\left|D^{\gamma} \varphi_{j}^{N}(\xi)\right| \leqslant c_{\gamma}\left(1+|\xi|^{2}\right)^{-\gamma / 2} \text { for any } \xi \in \mathbb{R}^{n} ;
$$

(iv) there exists a constant $c_{\varphi}>0$ such that

$$
0<\sum_{j=0}^{\infty} \varphi_{j}^{N}(\xi)=c_{\varphi}<\infty \quad \text { for any } \xi \in \mathbb{R}^{n} .
$$

For $\varphi \in \mathcal{S}\left(\mathbb{R}^{n}\right)$ and $f \in \mathcal{S}^{\prime}\left(\mathbb{R}^{n}\right)$ we will use the notation $\varphi(D) f(x)=$ $\left[\mathcal{F}^{-1}(\varphi \mathcal{F} f)\right](x)$, where $\mathcal{F}$ and $\mathcal{F}^{-1}$ stand, respectively, for the Fourier and inverse Fourier transform.

If $\left(f_{j}\right)_{j \in \mathbb{N}_{0}}$ is a sequence of complex-valued Lebesgue measurable functions on $\mathbb{R}^{n}$, then

$$
\left\|\left(f_{j}\right)_{j \in \mathbb{N}_{0}} \mid \ell_{q}\left(L_{p}\right)\right\|:=\left(\sum_{j=0}^{\infty}\left\|f_{j} \mid L_{p}\right\|^{q}\right)^{\frac{1}{q}}
$$

with appropriate modification if $q=\infty$.

Definition 3.2. Let $\left(\sigma_{j}\right)_{j \in \mathbb{N}_{0}}$ be an admissible sequence. Let $\left(N_{j}\right)_{j \in \mathbb{N}_{0}}$ be an admissible sequence satisfying Assumption 2 and let $\varphi^{N}$ be a system of functions as in Definition 3.1. Let $0<p \leqslant \infty$ and $0<q \leqslant \infty$. The Besov space of generalized smoothness is

$$
B_{p, q}^{\sigma, N}:=\left\{f \in \mathcal{S}^{\prime}:\left\|f\left|B_{p, q}^{\sigma, N}\|:=\|\left(\sigma_{j} \varphi_{j}^{N}(D) f\right)_{j \in \mathbb{N}_{0}}\right| \ell_{q}\left(L_{p}\right)\right\|<\infty\right\} .
$$

Remark 3.3. Note that if $0<p<\infty$ and $0<q \leqslant \infty$, then the TriebelLizorkin space of generalized smoothness $F_{p, q}^{\sigma, N}$ is defined in an analogous way, by interchanging the roles of the quasi-norms in $L_{p}$ and in $\ell_{q}$.

In order to keep this work at a reasonable length we decided to shift the problem concerning envelopes for $F$-spaces to a later paper.

One should note that if $1<p<\infty$ one can consider a weaker assumption on the sequence $N$ in the above definition, namely $\lambda_{0} N_{j} \leqslant N_{j+1}, \lambda_{0}>1$ (for any $j \in \mathbb{N}_{0}$ ), as discussed in [7]. 
The main tool in defining function spaces of generalized smoothness of Besov and Triebel-Lizorkin type for $1<p<\infty$ is the classical Fourier-multiplier theorem of Michlin-Hörmander type stated in [30, Theorem 2.2.4].

To extend the definition of the spaces of generalized smoothness to $p=\infty$, $p=1$, and to $0<p<1$, the assumption $N_{j+1} \leqslant \lambda_{1} N_{j}$ (for any $j \in \mathbb{N}_{0}$ ) is necessary. The reason is, that we cannot use in these cases the above mentioned Fourier-multiplier theorem. A substitute of it is a Fourier-multiplier theorem which was proved in spaces of entire analytic functions with the help of maximal functions, see $[31$, Theorems 1.6.2, 1.6.3]. These facts are discussed in [7, Section 3].

Remark 3.4. Note that if $N_{j}=2^{j}$ and $\sigma=\sigma^{s}=\left(2^{j s}\right)_{j \in \mathbb{N}_{0}}$ with $s$ real, then the above spaces coincide with the usual Besov spaces $B_{p, q}^{s}$ on $\mathbb{R}^{n}$.

These scales of spaces, together with the Triebel-Lizorkin spaces $F_{p, q}^{s}$, include many well-known function spaces and were systematically treated in the books of H. Triebel, see [30], [31], [32] and [33] and the references therein. Further background material can be found in the books of D. E. Edmunds and H. Triebel, see [6] and of T. Runst and W. Sickel, see [28], books in which the theory is complemented by several other aspects (entropy numbers, nonlinear partial differential equations, etc.).

Remark 3.5. The $B_{p, q}^{\sigma, N}$ are quasi-Banach spaces which are independent of the choice of the system $\left(\varphi_{j}^{N}\right)_{j \in \mathbb{N}_{0}}$, in the sense of equivalent quasi-norms (and this is the reason why we may omit in our notation reference to $\left.\left(\varphi_{j}^{N}\right)_{j \in \mathbb{N}_{0}}\right)$.

Remark 3.6. As in the classical case, compare [30, Theorem 2.3.2] or [31, Proposition 2.3.3], the embeddings $\mathcal{S} \hookrightarrow B_{p, q}^{\sigma, N} \hookrightarrow \mathcal{S}^{\prime}$ hold true for all admissible values of the parameters and sequences. If $p, q<\infty$, then $\mathcal{S}$ is dense in $B_{p, q}^{\sigma, N}$.

If $1<p<\infty$ and $1<q<\infty$ many results are already known from the works of G. A. Kalyabin and M. L. Goldman, see for example [10] - [16] and $[20]-[25]$.

It was shown in [7, Section 3.3] that the function spaces considered so far in this work cover (besides the classical Besov spaces $B_{p, q}^{s}$ ) many other classes of function spaces of generalized smoothness of Besov type known in the literature.

3.2. Embeddings. In what follows we will present some embedding results which will be useful when discussing envelopes for generalized smoothness but which are also of independent interest for the general theory of these spaces. We start fixing the notation.

Notation 1. We will use the convention $\frac{1}{\infty}=0$. If $r$ is a real number then $r_{+}=\max (r, 0)$. If $0<r \leqslant \infty$, then $r^{\prime}$ is given by $\frac{1}{r^{\prime}}=\left(1-\frac{1}{r}\right)_{+}$. In particular if $0<r \leqslant 1$, then $r^{\prime}=\infty$. 
Theorem 3.7. Let $N=\left(N_{j}\right)_{j \in \mathbb{N}_{0}}$ be an admissible sequence as in Assumption 2 and let $\sigma=\left(\sigma_{j}\right)_{j \in \mathbb{N}_{0}}$ and $\tau=\left(\tau_{j}\right)_{j \in \mathbb{N}_{0}}$ be two further admissible sequences. Let $0<p_{1} \leqslant p_{2} \leqslant \infty, 0<q_{1}, q_{2} \leqslant \infty$ and $\frac{1}{q^{*}}:=\left(\frac{1}{q_{2}}-\frac{1}{q_{1}}\right)_{+}$. If

$$
\left(\sigma_{j}^{-1} \tau_{j} N_{j}^{n\left(\frac{1}{p_{1}}-\frac{1}{p_{2}}\right)}\right)_{j \in \mathbb{N}_{0}} \in \ell_{q^{*}},
$$

then $\quad B_{p_{1}, q_{1}}^{\sigma, N} \hookrightarrow B_{p_{2}, q_{2}}^{\tau, N}$.

The ideas used in a proof of such result have been around for quite some time (see, for example, [26, Proposition 1.1.13])

Remark 3.8. Note that if $N_{j}=2^{j}, \sigma_{j}=2^{j s_{1}}$ and $\tau_{j}=2^{j s_{2}}\left(j \in \mathbb{N}_{0}\right)$ then from the above theorem we immediately get:

$$
\begin{aligned}
& \text { if } 0<q_{1} \leqslant q_{2} \leqslant \infty \text { and if } s_{1}-\frac{n}{p_{1}} \geqslant s_{2}-\frac{n}{p_{2}} \text {, then (classical spaces) } \\
& B_{p_{1}, q_{1}}^{s_{1}} \hookrightarrow B_{p_{2}, q_{2}}^{s_{2}} ; \\
& \text { if } 0<q_{2}<q_{1} \leqslant \infty \text { and if } s_{1}-\frac{n}{p_{1}}>s_{2}-\frac{n}{p_{2}} \text {, then (classical spaces) } \\
& B_{p_{1}, q_{1}}^{s_{1}} \hookrightarrow B_{p_{2}, q_{2}}^{s_{2}} .
\end{aligned}
$$

These results are well-known, see for example [31, Theorem 2.7.1 and Proposition 2.3.2/2]. For a forerunner of our general result in the above theorem one should see also [26, Proposition 1.1.13] .

Remark 3.9. Let $N=\left(N_{j}\right)_{j \in \mathbb{N}_{0}}$ be an admissible sequence as in Assumption 2 and let $0<p \leqslant \infty$ and $0<q \leqslant \infty$. Recall that in [7, Theorem 3.1.7] it was proved that we have the following identity at the level of zero smoothness:

$$
B_{p, q}^{\sigma^{0}, N}=B_{p, q}^{0}, \quad \text { where } \sigma^{0}=(1)_{j \in \mathbb{N}_{0}} .
$$

In fact (3.3) was stated in [7] only for $1<p<\infty, 1 \leqslant q \leqslant \infty$, but it is easy to see, following the lines of the proof of Theorem 3.1.7 in [7], that the identity holds for all $0<p, q \leqslant \infty$.

As a simple consequence of Theorem 3.7, (3.3) and [31, Proposition 2.5.7], we get the following.

Corollary 3.10. Let $N=\left(N_{j}\right)_{j \in \mathbb{N}_{0}}$ be an admissible sequence with $\lambda_{0}>1$ in (3.1) and let $\sigma=\left(\sigma_{j}\right)_{j \in \mathbb{N}_{0}}$ be an admissible sequence. Let $0<p \leqslant \infty$ and $0<q \leqslant \infty$. If

$$
\left(\sigma_{j}^{-1} N_{j}^{\frac{n}{p}}\right)_{j \in \mathbb{N}_{0}} \in \ell_{q^{\prime}}, \quad \text { then } \quad B_{p, q}^{\sigma, N} \hookrightarrow C,
$$

where here $C$ stands for the space of complex-valued, bounded and uniformly continuous functions on $\mathbb{R}^{n}$ endowed with the sup-norm.

Remark 3.11. Actually we really have an equivalence in this result, as will be shown later as a consequence of our results on growth envelope functions. If $1<p, q<\infty$ such an equivalence was first proved by G. A. Kalyabin in [24]. 
3.3. Atomic decompositions. An important tool in characterising function spaces of generalized smoothness is the atomic decomposition theorem. Following [7, Section 4.4] we will state here this theorem for Besov spaces of generalized smoothness.

Recall that the sequence $N$ is always as in Assumption 2. Let $\mathbb{Z}^{n}$ be the lattice of all points in $\mathbb{R}^{n}$ with integer-valued components. If $j \in \mathbb{N}_{0}$ and $m=\left(m_{1}, \ldots, m_{n}\right) \in \mathbb{Z}^{n}$ we denote $Q_{j m}$ the cube in $\mathbb{R}^{n}$ centred at $N_{j}^{-1} m=$ $\left(N_{j}^{-1} m_{1}, \ldots, N_{j}^{-1} m_{n}\right)$ which has sides parallel to the axes and side length $N_{j}^{-1}$. If $Q_{j m}$ is such a cube in $\mathbb{R}^{n}$ and $c>0$ then $c Q_{j m}$ is the cube in $\mathbb{R}^{n}$ concentric with $Q_{j m}$ and with side length $c N_{j}^{-1}$.

We are now prepared to introduce the $N$-atoms (associated to the sequence $N)$.

\section{Definition 3.12.}

(i) Let $M \in \mathbb{N}_{0}, c^{*}>1$. A function $a: \mathbb{R}^{n} \rightarrow \mathbb{C}$ which is $M$ times differentiable (continuous if $M=0$ ) is called an $1_{M}-N$-atom if

$$
\begin{gathered}
\text { supp } a \subset c^{*} Q_{0 m} \quad \text { for some } m \in \mathbb{Z}^{n} \\
\left|D^{\alpha} a(x)\right| \leqslant 1, \quad \text { if }|\alpha| \leqslant M .
\end{gathered}
$$

(ii) Let $\sigma=\left(\sigma_{j}\right)_{j \in \mathbb{N}_{0}}$ be an admissible sequence, let $0<p \leqslant \infty, M, L+1 \in \mathbb{N}_{0}$, $c^{*}>1$. A function $a: \mathbb{R}^{n} \rightarrow \mathbb{C}$ which is $M$ times differentiable (continuous if $M=0)$ is called an $(\sigma, p)_{M, L}-N$-atom if

$$
\begin{gathered}
\text { supp } a \subset c^{*} Q_{j m} \quad \text { for some } j \in \mathbb{N}, m \in \mathbb{Z}^{n} \\
\left|D^{\alpha} a(x)\right| \leqslant \sigma_{j}^{-1} N_{j}^{\frac{n}{p}+|\alpha|}, \quad \text { if }|\alpha| \leqslant M \\
\int_{\mathbb{R}^{n}} x^{\gamma} a(x) d x=0, \quad \text { if }|\gamma| \leqslant L .
\end{gathered}
$$

If the atom $a$ is located at $Q_{j m}$ (that means supp $a \subset c^{*} Q_{j m}$ with $j \in \mathbb{N}_{0}$, $\left.m \in \mathbb{Z}^{n}, c^{*}>1\right)$, then we will denote it by $a_{j m}$.

This concept generalises the smooth (isotropic) atoms from the works of M. Frazier and B. Jawerth, [8] and [9], which correspond to $N_{j}=2^{j}$ and $\sigma_{j}=2^{j s}$ with real $s$.

We give some technical explanations. The value of the number $c^{*}>1$ in (3.4) and (3.6) is unimportant (but it should be kept fixed). It simply makes clear that at the level $j$ some controlled overlapping of the supports of $a_{j m}$ must be allowed.

The moment conditions (3.8) can be reformulated as $D^{\gamma} \widehat{a}(0)=0$ if $|\gamma| \leqslant L$, which shows that a sufficiently strong decay of $\widehat{a}$ at the origin is required. If $L<0$, then (3.8) simply means that there are no moment conditions. 
The reason for the normalising factor in (3.5) and (3.7) is that in this way there exists a constant $c>0$ such that for all these atoms we have $\left\|a \mid B_{p, q}^{\sigma, N}\right\| \leqslant c$. Hence, as in the classical case, atoms are normalised building blocks satisfying some moment conditions.

Before we state the atomic decomposition theorem we have to introduce the sequence space $b_{p, q}$.

Definition 3.13. Let $0<p \leqslant \infty, 0<q \leqslant \infty$. Then $b_{p, q}$ is the collection of all sequences $\lambda=\left\{\lambda_{j m} \in \mathbb{C}: j \in \mathbb{N}_{0}, m \in \mathbb{Z}^{n}\right\}$ such that

$$
\left\|\lambda \mid b_{p, q}\right\|=\left(\sum_{j=0}^{\infty}\left(\sum_{m \in \mathbb{Z}^{n}}\left|\lambda_{j m}\right|^{p}\right)^{\frac{q}{p}}\right)^{\frac{1}{q}}
$$

(with the usual modification if $p=\infty$ and/or $q=\infty$ ) is finite.

Theorem 3.14. Let $N=\left(N_{j}\right)_{j \in \mathbb{N}_{0}}$ be an admissible sequence as in Assumption 2. Let $\sigma=\left(\sigma_{j}\right)_{j \in \mathbb{N}_{0}}$ be an admissible sequence with $\kappa_{0}, \kappa_{1}$ the corresponding constants, as in (2.1). Let $0<p \leqslant \infty, 0<q \leqslant \infty$, and let $M, L+1 \in \mathbb{N}_{0}$ be such that

$$
\begin{aligned}
M & >\frac{\log _{2} \kappa_{1}}{\log _{2} \lambda_{0}} \\
L & >-1+n\left(\frac{\log _{2} \lambda_{1}}{\log _{2} \lambda_{0}} \frac{1}{\min (1, p)}-1\right)-\frac{\log _{2} \kappa_{0}}{\log _{2} \lambda_{0}} .
\end{aligned}
$$

Let $c^{*}>1$ be fixed as in Definition 3.12. Then $f \in \mathcal{S}^{\prime}$ belongs to $B_{p, q}^{\sigma, N}$ if, and only if, it can be represented as

$$
f=\sum_{j=0}^{\infty} \sum_{m \in \mathbb{Z}^{n}} \lambda_{j m} a_{j m},
$$

convergence being in $\mathcal{S}^{\prime}$, where $a_{j m}$ are $1_{M}-N$-atoms $(j=0)$ or $(\sigma, p)_{M, L}-N$ atoms $(j \in \mathbb{N})$ and $\lambda \in b_{p, q}$, where $\lambda=\left\{\lambda_{j m}: j \in \mathbb{N}_{0}, m \in \mathbb{Z}^{n}\right\}$. Furthermore, inf $\left\|\lambda \mid b_{p, q}\right\|$, where the infimum is taken over all admissible representations (3.11), is an equivalent quasi-norm in $B_{p, q}^{\sigma, N}$.

For further comments, remarks, examples related to the above theorem we refer the interested reader to [7].

3.4. Rearrangement properties and embeddings in $L_{1}^{\text {loc }}$. If $f$ is an extended complex-valued measurable function on $\mathbb{R}^{n}$ which is finite a.e., then the decreasing rearrangement of $f$ is the function defined on $[0, \infty)$ by

$$
f^{*}(t):=\inf \left\{\lambda \geqslant 0: m_{f}(\lambda) \leqslant t\right\}, \quad t \geqslant 0
$$


with $m_{f}$ being the distribution function given by

$$
m_{f}(\lambda):=\left|\left\{x \in \mathbb{R}^{n}:|f(x)|>\lambda\right\}\right|, \quad \lambda \geqslant 0 .
$$

As usual, the convention $\inf \emptyset=\infty$ is assumed and $|\cdot|$ denotes the Lebesgue measure when applied to measurable subsets of $\mathbb{R}^{n}$. Moreover, the maximal function of $f^{*}$ is the function

$$
f^{* *}(t):=\frac{1}{t} \int_{0}^{t} f^{*}(\tau) d \tau, \quad t>0 .
$$

We assume that the reader is familiar with basic facts concerning rearrangements. These may be found in [1], for example. In particular we shall need the sub-additivity property

$$
(f+g)^{* *}(t) \leqslant f^{* *}(t)+g^{* *}(t), \quad t>0 .
$$

By analogy, in the case of a (multiple) sequence $\left(\alpha_{m}\right)_{m \in \mathbb{Z}^{n}} \subset \mathbb{C}$, its decreasing rearrangement is defined as the sequence $\left(\alpha_{l}^{*}\right)_{l \in \mathbb{N}}$, where

$$
\alpha_{l}^{*}:=\inf \left\{\lambda \geqslant 0: \#\left\{m \in \mathbb{Z}^{n}:\left|\alpha_{m}\right|>\lambda\right\}<l\right\}, \quad l \in \mathbb{N} .
$$

We also define

$$
\alpha_{l}^{* *}:=\frac{1}{l} \sum_{k=1}^{l} \alpha_{k}^{*}, \quad l \in \mathbb{N} .
$$

Proposition 3.15. Let $p \in(1, \infty]$. Let $\left(\alpha_{m}\right)_{m \in \mathbb{Z}^{n}},\left(\alpha_{l}^{*}\right)_{l \in \mathbb{N}}$ and $\left(\alpha_{l}^{* *}\right)_{l \in \mathbb{N}}$ be as above. Then

$$
\left\|\left(\alpha_{m}\right)_{m \in \mathbb{Z}^{n}}\left|\ell_{p}\|=\|\left(\alpha_{l}^{*}\right)_{l \in \mathbb{N}}\right| \ell_{p}\right\| \leqslant\left\|\left(\alpha_{l}^{* *}\right)_{l \in \mathbb{N}}\left|\ell_{p}\left\|\leqslant \frac{p}{p-1}\right\|\left(\alpha_{l}^{*}\right)_{l \in \mathbb{N}}\right| \ell_{p}\right\|,
$$

where $\frac{p}{p-1}$ should be interpreted as 1 when $p=\infty$.

Proof. The result is obvious for $p=\infty$. As to the case $p \in(1, \infty)$, the equality (which, actually, holds also for $0<p \leqslant 1$ ) follows from [1, Proposition 1.8 in Chapter 2, p. 43] applied to the counting measure in $\mathbb{Z}^{n}$ and the last inequality is due to Hardy and Landau [18, pp. 239-240].

Recall that whenever a sequence $N$ is considered, Assumption 2 is implied. The following lemma is an analogue to [26, Lemma 1.3.6]. Though it has an elementary proof, it plays a key role in proving the next proposition.

Lemma 3.16. Let $c^{*}>1$ and $x \in \mathbb{R}^{n}$. Given a $j \in \mathbb{N}_{0}$ then $x$ belongs to at most $K$ cubes $c^{*} Q_{j m}, m \in \mathbb{Z}^{n}$, where $K$ does not depend on $j$ and $m$ (but may depend on $c^{*}$ and on the dimension $\left.n\right)$. 
The proof of next proposition is similar (up to the last part) to the proof of Proposition 3.7 in [5], so we decided to omit it too. Recall $q^{\prime}$ is the conjugate of $q$ (with $q^{\prime}=\infty$ when $0<q \leqslant 1$ ).

Proposition 3.17. Let $c^{*}>1$ and $\left(d_{j}\right)_{j \in \mathbb{N}_{0}}$ be a sequence of positive numbers. Let $\left(a_{j m}\right)_{j \in \mathbb{N}_{0}, m \in \mathbb{Z}^{n}}$ be a sequence of complex-valued measurable functions on $\mathbb{R}^{n}$ such that, for each $j$ and $m, \operatorname{supp} a_{j m} \subset c^{*} Q_{j m}$ and $\left|a_{j m}(x)\right| \leqslant d_{j}$, for all $x \in \mathbb{R}^{n}$, where $Q_{j m}$ is a cube as defined in the previous subsection. Let $\left(\lambda_{j m}\right)_{j \in \mathbb{N}_{0}, m \in \mathbb{Z}^{n}}$ be a sequence of complex numbers and define, for any $j \in \mathbb{N}_{0}$,

$$
f_{j}(x):=\sum_{m \in \mathbb{Z}^{n}} \lambda_{j m} a_{j m}(x), \quad x \in \mathbb{R}^{n} .
$$

(i) There are positive constants $C$ and $D$, depending only on $n$ and $c^{*}$, such that

$$
\begin{gathered}
f_{j}^{*}(t) \leqslant D d_{j} \sum_{l=1}^{\infty} \lambda_{j l}^{*} \chi_{j l}(t), \quad t \geqslant 0, j \in \mathbb{N}_{0} \\
f_{j}^{* *}(t) \leqslant D d_{j} \sum_{l=1}^{\infty} \lambda_{j l}^{* *} \chi_{j l}(t), \quad t>0, j \in \mathbb{N}_{0},
\end{gathered}
$$

where $\chi_{j l}$ is the characteristic function of the set $\left[C N_{j}^{-n}(l-1), C N_{j}^{-n} l\right)$, $l \in \mathbb{N},\left(\lambda_{j l}^{*}\right)_{l \in \mathbb{N}}$ is the decreasing rearrangement of $\left(\lambda_{j m}\right)_{m \in \mathbb{Z}^{n}}, j \in \mathbb{N}_{0}$, and $\lambda_{j l}^{* *}:=\frac{1}{l} \sum_{k=1}^{l} \lambda_{j k}^{*}, l \in \mathbb{N}$.

(ii) If, for some $j \in \mathbb{N}_{0}$ and $0<p \leqslant \infty$, we have $\left(\lambda_{j m}\right)_{m \in \mathbb{Z}^{n}} \in \ell_{p}\left(\mathbb{Z}^{n}\right)$, then $f_{j} \in L_{p}$, for the same $j$ and $p$.

(iii) Let $0<p \leqslant \infty, 0<q \leqslant \infty$ and assume $\left(\lambda_{j m}\right)_{j \in \mathbb{N}_{0}, m \in \mathbb{Z}^{n}} \in b_{p, q}$, where $b_{p, q}$ was introduced in Definition 3.13. If, moreover,

$$
\left(d_{j} \cdot N_{j}^{-\frac{n}{\max (1, p)}}\right)_{j \in \mathbb{N}_{0}} \in \ell_{q^{\prime}}
$$

then the series $\sum_{j=0}^{\infty} f_{j}$ converges in $L_{\max (1, p)}$ to a function $f$ satisfying

$$
f^{* *}(t) \leqslant \sum_{j=0}^{\infty} f_{j}^{* *}(t), \quad t>0 .
$$

Corollary 3.18. Let $N=\left(N_{j}\right)_{j \in \mathbb{N}_{0}}$ be an admissible sequence with $\lambda_{0}>1$ in (3.1) and let $\sigma=\left(\sigma_{j}\right)_{j \in \mathbb{N}_{0}}$ be an admissible sequence. Let $0<p \leqslant \infty$ and $0<q \leqslant \infty$. If

$$
\left(\sigma_{j}^{-1} N_{j}^{n\left(\frac{1}{p}-1\right)_{+}}\right)_{j \in \mathbb{N}_{0}} \in \ell_{q^{\prime}}
$$

where $q^{\prime}$ is conjugate to $q$ (with $q^{\prime}=\infty$ when $0<q \leqslant 1$ ), then $B_{p, q}^{\sigma, N} \hookrightarrow L_{\max (1, p)}$. 
Proof. From the atomic decomposition theorem (Theorem 3.14) we know that any $f \in B_{p, q}^{\sigma, N}$ is an infinite sum, in $\mathcal{S}^{\prime}$, of $f_{n}{ }_{j}$ 's as in Proposition 3.17, where $\left(\lambda_{j m}\right)_{j \in \mathbb{N}_{0}, m \in \mathbb{Z}^{n}} \in b_{p, q}, d_{0}=1$ and $d_{j}=\sigma_{j}^{-1} N_{j}^{\frac{n}{p}}$ for $j \in \mathbb{N}$. Since (3.14) is in this case equivalent to (3.15), part (iii) of Proposition 3.17 guarantees that $\sum_{j=0}^{\infty} f_{j}$ also converges to $f$ in $L_{\max (1, p)}$.

Remark 3.19. Note that if $1<p<\infty$ and $1<q<\infty$ the above result says that if the sequence $\left(\sigma_{j}\right)_{j \in \mathbb{N}_{0}}$ has additionally the property $\left(\sigma_{j}^{-1}\right)_{j \in \mathbb{N}_{0}} \in l_{q^{\prime}}$, then all elements of $B_{p, q}^{\sigma, N}$ are at least functions in $L_{p}$. This fact is well known from the earlier works of M. L. Goldman and G. A. Kalyabin. Our Corollary 3.18 is the natural generalisation of these results.

Remark 3.20. As an immediate consequence we get that if $\sigma, N, p$ and $q$ are as in Corollary 3.18 and if (3.15) is satisfied, then $B_{p, q}^{\sigma, N} \subset L_{1}^{\text {loc }}$.

\section{Local growth envelopes for $B_{p, q}^{\sigma, N}$}

4.1. Fundamentals about envelopes. As we have briefly mentioned in the Introduction, regarding the study of local growth envelopes in the context of the spaces $B_{p, q}^{\sigma, N}$, of interest are the spaces so that

$$
B_{p, q}^{\sigma, N} \subset L_{1}^{\text {loc }} \quad \text { but } \quad B_{p, q}^{\sigma, N} \nrightarrow L_{\infty}
$$

Assume $N=\left(N_{j}\right)_{j \in \mathbb{N}_{0}}$ and $\sigma=\left(\sigma_{j}\right)_{j \in \mathbb{N}_{0}}$ are admissible sequences and for the first one one has $\lambda_{0}>1$. As to the inclusion

$$
B_{p, q}^{\sigma, N} \subset L_{1}^{l o c}
$$

this is the case if

$$
\left(\sigma_{j}^{-1} N_{j}^{n\left(\frac{1}{p}-1\right)_{+}}\right)_{j \in \mathbb{N}_{0}} \in \ell_{q^{\prime}}
$$

as pointed out in Remark 3.20. Though the inclusion (4.1) may also occur if (4.2) is false, we really need that assumption - and even a little bit more, namely

$$
\left\{\begin{array}{cl}
\left(\underline{\sigma}_{j}^{-1}\right)_{j \in \mathbb{N}_{0}} \in \ell_{\min (q, 1)} & \text { if } p>1 \\
\left(\underline{\sigma}_{j}^{-1} \bar{N}_{j}^{n\left(\frac{1}{p}-1\right)+\delta}\right)_{j \in \mathbb{N}_{0}} \in \ell_{\min (q, 1)}, \text { for some } \delta>0, & \text { if } 0<p \leqslant 1
\end{array}\right.
$$

- in order to prove one of our main results (see Proposition 4.5 below).

Remark 4.1. Using $\min (q, 1) \leqslant q^{\prime}$ it immediately follows that (4.3) implies (4.2). 
On the other hand, we believe that, by assuming (4.3), we are "only" excluding what can be called "border" situations. In fact, in the classical case $N_{j}=2^{j}, \sigma_{j}=2^{j s}$, with $s \in \mathbb{R},(4.3)$ turns out to be equivalent to $s>n\left(\frac{1}{p}-1\right)_{+}$, and it is known that for $s<n\left(\frac{1}{p}-1\right)_{+}$the corresponding inclusion fails, while in the "borderline" $s=n\left(\frac{1}{p}-1\right)_{+}$one needs extra information on the parameters in order to distinguish between "inclusion" and "no inclusion" - see [29].

As to $B_{p, q}^{\sigma, N}$ not being continuously embedded in $L_{\infty}$, one knows from Corollary 3.10 that only

$$
\left(\sigma_{j}^{-1} N_{j}^{\frac{n}{p}}\right)_{j \in \mathbb{N}_{0}} \notin \ell_{q^{\prime}}
$$

is worth considering. Therefore, though the (extended real-valued, decreasing) function

$$
\mathcal{E}_{\mathrm{LG}} \mid B_{p, q}^{\sigma, N}(t):=\sup \left\{f^{*}(t):\left\|f \mid B_{p, q}^{\sigma, N}\right\| \leqslant 1\right\}, \quad t>0,
$$

which will help us measure the ability of local growth for functions in $B_{p, q}^{\sigma, N}$, makes perfectly good sense by merely assuming that $B_{p, q}^{\sigma, N} \subset L_{1}^{\text {loc }}$, we will tend to restrict our attention to the situations when the admissible sequences $\left(\sigma_{j}\right)_{j \in \mathbb{N}_{0}}$ and $\left(N_{j}\right)_{j \in \mathbb{N}_{0}}$ (this one with the further assumption $\lambda_{0}>1$ in (3.1)) satisfy (4.3) and (4.4). We can say, in such a case, that $\mathcal{E}_{\mathrm{LG}} \mid B_{p, q}^{\sigma, N}$ (which will then be finite for $t>0$, in view of Proposition 4.5 below) defines a decreasing function which is positive in $(0, \varepsilon]$, for some $\varepsilon \in(0,1)$, and which tends to $\infty$ as $t$ goes to 0 (see Proposition 4.8 below). Therefore, it will make sense to ask for the behaviour of $\mathcal{E}_{\mathrm{LG}} \mid B_{p, q}^{\sigma, N}(t)$ near zero, which will give an indication of the ability of local growth for functions in $B_{p, q}^{\sigma, N}$.

Let $\mathcal{E}_{\mathrm{LG}}$ be the set of all functions $f:(0, \varepsilon] \rightarrow \mathbb{R}^{+}$, for any $\varepsilon \in(0,1]$, which are decreasing and consider the following equivalence relation in $\mathcal{E}_{\mathrm{LG}}$ : given $f, g \in \mathcal{E}_{\mathrm{LG}}$, one says that $f$ and $g$ are equivalent (and write $f \sim_{\mathrm{LG}} g$ ) if

$$
\exists c_{1}, c_{2}>0: \forall t \in(0, \varepsilon], c_{1} g(t) \leqslant f(t) \leqslant c_{2} g(t),
$$

where $(0, \varepsilon]$ is the smallest of the domains of $f$ and $g$.

Definition 4.2. The local growth envelope function of $B_{p, q}^{\sigma, N}$, for $\sigma, p, q$ and $N$ as stated above, is the equivalence class $\left[\mathcal{E}_{\mathrm{LG}} \mid B_{p, q}^{\sigma, N}\right]$. We shall also call local growth envelope function of $B_{p, q}^{\sigma, N}$ any representative in such a class. We even call local growth envelope function of $B_{p, q}^{\sigma, N}$ any function $f:(0, \varepsilon] \rightarrow \mathbb{R}^{+}$, for some $\varepsilon \in(0,1]$, - even if not decreasing - such that $f \sim \mathcal{E}_{\mathrm{LG}} \mid B_{p, q}^{\sigma, N}$ in $(0, \varepsilon]$, and use it to represent the equivalence class $\left[\mathcal{E}_{\mathrm{LG}} \mid B_{p, q}^{\sigma, N}\right]$.

Remark 4.3. Note that different equivalent quasi-norms taken in the same space $B_{p, q}^{\sigma, N}$ give rise to the same equivalence class $\left[\mathcal{E}_{\mathrm{LG}} \mid B_{p, q}^{\sigma, N}\right]$.

Let again $\sigma, p, q$ and $N$ be as stated above. Assume there exists a continuous representative $\mathcal{E}_{\mathrm{LG}} B_{p, q}^{\sigma, N} \in\left[\mathcal{E}_{\mathrm{LG}} \mid B_{p, q}^{\sigma, N}\right]$ (we shall later see that this is indeed the 
case). Let $(0, \varepsilon], 0<\varepsilon<1$, be its domain. Define $H(t):=-\log _{2} \mathcal{E}_{\mathrm{LG}} B_{p, q}^{\sigma, N}(t)$ and note that $H$ is a (finite) real increasing function on $(0, \varepsilon]$ which tends to $-\infty$ when $t$ goes to 0 . There is only a Borel measure (i.e., a measure defined on the Borel sets) $\mu_{H}$ in $(0, \varepsilon]$ such that $\mu_{H}([a, b])=H(b)-H(a), \forall[a, b] \subset$ $(0, \varepsilon]$. Its restriction to each such $[a, b]$ is the Stieltjes-Borel measure associated with $\left.H\right|_{[a, b]}$.

In the important case when $H$ happens to be continuously differentiable in $(0, \varepsilon]$, we have $\mu_{H}(d t)=H^{\prime} d t$, and for the functions we want to integrate we can calculate the integrals as improper Riemann integrals.

Definition 4.4. Let $\sigma, p, q, N$ be as stated above. Then

$$
\mathfrak{E}_{\mathrm{LG}} B_{p, q}^{\sigma, N}:=\left(\left[\mathcal{E}_{\mathrm{LG}} \mid B_{p, q}^{\sigma, N}\right], u\right)
$$

is called the local growth envelope of $B_{p, q}^{\sigma, N}$, if $u$ is the minimum (assuming that it exists) of all $v>0$ such that

$$
\exists c(v)>0: \forall f \in B_{p, q}^{\sigma, N}, \quad\left(\int_{(0, \varepsilon]}\left(\frac{f^{*}(t)}{h(t)}\right)^{v} \mu_{H}(d t)\right)^{\frac{1}{v}} \leqslant c(v)\left\|f \mid B_{p, q}^{\sigma, N}\right\|,
$$

where $h(t)$ is a continuous representative in $\left[\mathcal{E}_{\mathrm{LG}} \mid B_{p, q}^{\sigma, N}\right]$ with domain $(0, \varepsilon]$, $0<\varepsilon<1$.

We must remark that this definition makes sense, namely that the infimum of all such $v$ 's is independent of the chosen continuous representative $h(t)$ in $\left[\mathcal{E}_{\mathrm{LG}} \mid B_{p, q}^{\sigma, N}\right]$, as follows by using some standard arguments of measure and integration theory, the definition of $\mathcal{E}_{\mathrm{LG}} \mid B_{p, q}^{\sigma, N}$ and [33, Proposition 12.2]. Recall, on the other hand, that we are assuming that there exists at least one such representative - and we have already mentioned that this is indeed the case, as will be apparent later. Recall also that the definition of $\mathcal{E}_{\mathrm{LG}} \mid B_{p, q}^{\sigma, N}$ guarantees that (4.5) holds at least for $v=\infty$. Remark also that the definition does not discard the possibility that there is no such thing called the local growth envelope of $B_{p, q}^{\sigma, N}$ : this would be the case if the infimum of the mentioned $v$ 's were not a minimum. We shall, however, see (in the following subsections) that the minimum is really attained, and therefore all mentioned spaces have local growth envelopes.

Instead of $\left(\left[\mathcal{E}_{\mathrm{LG}} \mid B_{p, q}^{\sigma, N}\right], u\right)$, we shall usually write $(h(t), u)$ for the local growth envelope of $B_{p, q}^{\sigma, N}$ with the assumptions made above, where $h(t)$ is any continuous representative in $\left[\mathcal{E}_{\mathrm{LG}} \mid B_{p, q}^{\sigma, N}\right]$. Instead of $h(t)$, we can also use in the couple any local growth envelope function as considered in Definition 4.2, though it must be borne in mind that for the construction of the measure $\mu_{H}$ we shall only use continuous representatives in $\left[\mathcal{E}_{\mathrm{LG}} \mid B_{p, q}^{\sigma, N}\right]$. 


\subsection{Estimates from above.}

Proposition 4.5. Let $0<p, q \leqslant \infty$. Let $\left(N_{j}\right)_{j \in \mathbb{N}_{0}}$ be a sequence as in Assumption 2 and $\left(\sigma_{j}\right)_{j \in \mathbb{N}_{0}}$ be an admissible sequence. Assume that

$$
\left(\sigma_{j}^{-1} N_{j}^{\frac{n}{p}}\right)_{j \in \mathbb{N}_{0}} \notin \ell_{q^{\prime}}
$$

and (4.3) holds. Let $\Lambda$ be an admissible function such that $\Lambda(z) \sim \sigma_{j}, z \in$ $\left[N_{j}, N_{j+1}\right], j \in \mathbb{N}_{0}$, with equivalence constants independent of $j$, and let $\Phi_{q^{\prime}}$, defined in $\left(0, N_{J_{0}}^{-n}\right]$, be the function from Definition 2.4 (with $q^{\prime}$ instead of $u$ ), where $J_{0} \in \mathbb{N}$ is chosen such that $N_{J_{0}}>1$. Then there exists an $\varepsilon \in(0,1)$ and $c>0$ such that

$$
\mathcal{E}_{\mathrm{LG}} \mid B_{p, q}^{\sigma, N}(t) \leqslant c \Phi_{q^{\prime}}(t), \quad \text { for any } t \in(0, \varepsilon],
$$

and, for each $v \in[q, \infty]$, there exists $c(v)>0$ such that

$$
\left(\int_{0}^{\varepsilon}\left(\frac{f^{*}(t)}{\Phi_{q^{\prime}}(t)}\right)^{v} \mu_{q^{\prime}}(d t)\right)^{\frac{1}{v}} \leqslant c(v)\left\|f \mid B_{p, q}^{\sigma, N}\right\|, \quad \text { for any } f \in B_{p, q}^{\sigma, N},
$$

with the modification

$$
\sup _{0<t \leqslant \varepsilon} \frac{f^{*}(t)}{\Phi_{q^{\prime}}(t)} \leqslant c(v)\left\|f \mid B_{p, q}^{\sigma, N}\right\| \quad \text { if } v=\infty
$$

where $\mu_{q^{\prime}}$ denotes the Borel measure associated with $-\log _{2} \Phi_{q^{\prime}}$ in $(0, \varepsilon]$ (in accordance with Subsection 4.1).

Proof. We will omit the details of calculations which are similar to the ones used in the proof of [5, Proposition 4.1]. First note that the hypotheses imply that $p \neq \infty$ and $B_{p, q}^{\sigma, N} \subset L_{1}^{\text {loc }}$. Fix $c^{*}>1$ as in Proposition 3.17 and consider the corresponding constants $C$ and $D$. Define $\varepsilon:=C N_{k_{0}}^{-n} \leqslant N_{J_{0}}^{-n}$, for a suitable chosen $k_{0} \in \mathbb{N}$.

Step 1: Here we assume $1<p<\infty$ and $q=\infty$ and we prove (4.6) and the modified version (4.8) of (4.7).

Given $f \in B_{p, \infty}^{\sigma, N}$ consider a corresponding atomic decomposition $\sum_{j=0}^{\infty} f_{j}$ (convergence in $\mathcal{S}^{\prime}$ ), where $f_{j}$ have the same meaning as in (3.12), for given atoms $a_{j m}$ in $B_{p \infty}^{\sigma, N}$ and complex numbers $\lambda_{j m}$ satisfying $\left(\lambda_{j m}\right)_{j \in \mathbb{N}_{0}, m \in \mathbb{Z}^{n}} \in b_{p, \infty}$. Together with our hypotheses, this guarantees that Proposition 3.17 can be applied with $d_{0}=1, d_{j}:=\sigma_{j}^{-1} N_{j}^{\frac{n}{p}}, j \in \mathbb{N}$. In particular, $f$ is also the limit, in $L_{p}$, of the series $\sum_{j=0}^{\infty} f_{j}$. This justifies the following inequality:

$$
\sup _{0<t \leqslant \varepsilon} \frac{f^{*}(t)}{\Phi_{1}(t)} \leqslant \sup _{k \geqslant k_{0}}\left(\frac{\sum_{j=0}^{k} f_{j}^{* *}\left(C N_{k+1}^{-n}\right)}{\Phi_{1}\left(C N_{k}^{-n}\right)}+\frac{\sum_{j=k+1}^{\infty} f_{j}^{* *}\left(C N_{k+1}^{-n}\right)}{\Phi_{1}\left(C N_{k}^{-n}\right)}\right) .
$$


Since, for $0 \leqslant j \leqslant k, C N_{k+1}^{-n} \in\left(0, C N_{j}^{-n}\right)$, then (3.13), Lemma 2.6, Proposition 2.7, Remark 2.8 and Proposition 3.15 allow us to write

$$
\frac{\sum_{j=0}^{k} f_{j}^{* *}\left(C N_{k+1}^{-n}\right)}{\Phi_{1}\left(C N_{k}^{-n}\right)} \leqslant c_{1} \frac{\sum_{j=0}^{k} \sigma_{j}^{-1} N_{j}^{\frac{n}{p}} \cdot \lambda_{j 1}^{*}}{\sum_{j=0}^{k} \sigma_{j}^{-1} N_{j}^{\frac{n}{p}}} \leqslant c_{1}\left\|\left(\lambda_{j m}\right)_{j \in \mathbb{N}_{0}, m \in \mathbb{Z}^{n}} \mid b_{p, \infty}\right\|,
$$

where $c_{1}>0$ is independent of $f$ and the atomic decomposition taken. For $j \geqslant k+1$ we have $C N_{k+1}^{-n} \in\left[C N_{j}^{-n}\left[N_{j}^{n} N_{k+1}^{-n}\right], C N_{j}^{-n}\left(\left[N_{j}^{n} N_{k+1}^{-n}\right]+1\right)\right)$ and by Proposition 3.17 with $l=\left[N_{j}^{n} N_{k+1}^{-n}\right]+1$ we have

$$
\sum_{j=k+1}^{\infty} f_{j}^{* *}\left(C N_{k+1}^{-n}\right) \leqslant c_{1} \sum_{j=k+1}^{\infty} \sigma_{j}^{-1} N_{j}^{\frac{n}{p}} \cdot \lambda_{j,\left[N_{j}^{n} N_{k+1}^{-n}\right]+1}^{* *}
$$

Decomposing

$$
\sum_{l=1}^{\infty} \lambda_{j l}^{* * p}=\sum_{m=0}^{\infty} \sum_{h=\left[N_{m}^{n} N_{k+1}^{-n}\right]+1}^{\left[N_{m+1}^{n} N_{k+1}^{-n}\right]} \lambda_{j h}^{* * p}
$$

where the inner sum is zero if $\left[N_{m}^{n} N_{k+1}^{-n}\right]+1>\left[N_{m+1}^{n} N_{k+1}^{-n}\right]$, we get

$$
\begin{aligned}
\sum_{l=1}^{\infty} \lambda_{j l}^{* * p} & \geqslant \sum_{m=0}^{\infty}\left(\left[N_{m+1}^{n} N_{k+1}^{-n}\right]-\left[N_{m}^{n} N_{k+1}^{-n}\right]\right) \lambda_{j,\left[N_{m+1}^{n} N_{k+1}^{-n}\right]}^{* * p} \\
& \geqslant\left(\left[N_{j}^{n} N_{k+1}^{-n}\right]-\left[N_{j-1}^{n} N_{k+1}^{-n}\right]\right) \lambda_{j,\left[N_{j}^{n} N_{k+1}^{-n}\right]+1}^{* * p}
\end{aligned}
$$

Let $\alpha$ be the smallest natural number such that

$$
\lambda_{0}^{n \alpha}>\frac{1}{\lambda_{0}^{n}-1}
$$

Clearly if $j \geqslant k+\alpha+2$ one has $N_{j-1}^{n} N_{k+1}^{-n} \geqslant \lambda_{0}^{n \alpha}$. So for any $j$ and $k$ with $j \geqslant k+\alpha+2$ we have $\left[N_{j}^{n} N_{k+1}^{-n}\right]-\left[N_{j-1}^{n} N_{k+1}^{-n}\right] \geqslant\left(\lambda_{0}^{n}-1-\lambda_{0}^{-n \alpha}\right) N_{j-1}^{n} N_{k+1}^{-n}$. Consequently, for any $j$ and $k$ with $j \geqslant k+\alpha+2$ we have

$$
\sum_{l=1}^{\infty} \lambda_{j l}^{* * p} \geqslant c_{2} N_{j-1}^{n} N_{k+1}^{-n} \lambda_{j,\left[N_{j}^{n} N_{k+1}^{-n}\right]+1}^{* * p}
$$

and this implies

$$
\lambda_{j,\left[N_{j}^{n} N_{k+1}^{-n}\right]+1}^{* *} \leqslant c_{3} N_{j}^{-\frac{n}{p}} N_{k}^{\frac{n}{p}}\left(\sum_{l=1}^{\infty} \lambda_{j l}^{* * p}\right)^{\frac{1}{p}} \quad \text { for any } j \geqslant k+\alpha+2 .
$$


Moreover,

$$
\lambda_{j,\left[N_{j}^{n} N_{k+1}^{-n}\right]+1}^{* *} \leqslant \lambda_{j, 2}^{* *} \leqslant\left(\sum_{l=1}^{\infty} \lambda_{j l}^{* * p}\right)^{\frac{1}{p}} \quad \text { if } k+1 \leqslant j \leqslant k+\alpha+1 .
$$

Since for $k+1 \leqslant j \leqslant k+\alpha+1$ we have $N_{j} \sim N_{k}$ with equivalence constants independent of $j$ and $k$ (but depending on $\alpha$ ) we can see that (4.12) holds true for all $j \geqslant k+1$ up to a different constant $c_{3}$. So we can insert (4.12), for all $j \geqslant k+1$, in (4.11) and using Proposition 3.15 we get

$$
\begin{aligned}
\sum_{j=k+1}^{\infty} f_{j}^{* *}\left(C N_{k+1}^{-n}\right) & \leqslant c_{4} \sum_{j=k+1}^{\infty} \sigma_{j}^{-1} N_{k}^{\frac{n}{p}} \cdot\left\|\left(\lambda_{j l}^{* *}\right)_{l \in \mathbb{N}} \mid \ell_{p}\right\| \\
& \leqslant c_{4}\left(\sum_{j=k+1}^{\infty} \sigma_{j}^{-1} N_{k}^{\frac{n}{p}}\right) \cdot \sup _{j \in \mathbb{N}_{0}}\left\|\left(\lambda_{j m}\right)_{m \in \mathbb{Z}^{n}} \mid \ell_{p}\right\| .
\end{aligned}
$$

Then Lemma 2.6, Proposition 2.7, (3.13) and Proposition 3.15 allow us to write

$$
\begin{aligned}
\frac{\sum_{j=k+1}^{\infty} f_{j}^{* *}\left(C N_{k+1}^{-n}\right)}{\Phi_{1}\left(C N_{k}^{-n}\right)} & \leqslant c_{5} \frac{\sum_{j=k+1}^{\infty} \sigma_{j}^{-1} N_{k}^{\frac{n}{p}}}{\sum_{j=1}^{k} \sigma_{j}^{-1} N_{j}^{\frac{n}{p}}}\left\|\left(\lambda_{j m}\right)_{j \in \mathbb{N}_{0}, m \in \mathbb{Z}^{n}} \mid b_{p, \infty}\right\| \\
& \leqslant c_{5}\left(\sum_{j=1}^{\infty} \underline{\sigma}_{j}^{-1}\right)\left\|\left(\lambda_{j m}\right)_{j \in \mathbb{N}_{0}, m \in \mathbb{Z}^{n}} \mid b_{p, \infty}\right\|
\end{aligned}
$$

where the constants are independent of $f$ and the atomic decomposition taken and we recall notation (2.2).

Using our assumption (4.3) and putting (4.9), (4.10) and (4.13) together, we get

$$
\sup _{0<t \leqslant \varepsilon} \frac{f^{*}(t)}{\Phi_{1}(t)} \leqslant c_{6}\left\|\left(\lambda_{j m}\right)_{j \in \mathbb{N}_{0}, m \in \mathbb{Z}^{n}} \mid b_{p, \infty}\right\|<\infty,
$$

for some $c_{6}>0$ independent of $f$ and the atomic decomposition taken, and, with the help of Theorem 3.14,

$$
\sup _{0<t \leqslant \varepsilon} \frac{f^{*}(t)}{\Phi_{1}(t)} \leqslant c_{7}\left\|f \mid B_{p, \infty}^{\sigma, N}\right\| .
$$

From this it easily follows, in the case $p>1$ and $q=\infty$, that (4.6) holds and, in particular, that $\mathcal{E}_{\mathrm{LG}} \mid B_{p, \infty}^{\sigma, N}(t)$ is finite for each $t \in(0, \varepsilon]$.

Step 2: We assume now $1<p<\infty$ and $1<q<\infty$ and will prove (4.6) and (4.7).

We start with the proof of (4.7) when $v=q$. Given $f \in B_{p, q}^{\sigma, N}$ consider a corresponding atomic decomposition $\sum_{j=0}^{\infty} f_{j}$ (convergence in $\mathcal{S}^{\prime}$ ), where $f_{j}$ 
have the same meaning as in (3.12), for given atoms $a_{j m}$ in $B_{p, q}^{\sigma, N}$ and complex numbers $\lambda_{j m}$ satisfying $\left(\lambda_{j m}\right)_{j \in \mathbb{N}_{0}, m \in \mathbb{Z}^{n}} \in b_{p, q}$. Together with our hypotheses, this guarantees that Proposition 3.17 can be applied with $d_{0}=1, d_{j}:=\sigma_{j}^{-1} N_{j}^{\frac{n}{p}}$, $j \in \mathbb{N}$. In particular, $f$ is also the limit, in $L_{p}$, of the series $\sum_{j=0}^{\infty} f_{j}$.

Noting that for $C N_{k+1}^{-n} \leqslant t \leqslant C N_{k}^{-n}$ one has $\Lambda\left(t^{-\frac{1}{n}}\right) \sim \sigma_{k}$ we get using the properties of the measure $\mu_{q^{\prime}}$, namely Lemma 2.5 ,

$$
\begin{aligned}
& \left(\int_{0}^{\varepsilon}\left(\frac{f^{*}(t)}{\Phi_{q^{\prime}}(t)}\right)^{q} \mu_{q^{\prime}}(d t)\right)^{\frac{1}{q}} \\
& =c_{1}\left(\int_{0}^{\varepsilon} \frac{f^{*}(t)^{q}}{\Phi_{q^{\prime}}(t)^{q q^{\prime}}} \cdot \frac{1}{n q^{\prime}} t^{-\frac{q^{\prime}}{p}-1} \Lambda\left(t^{-\frac{1}{n}}\right)^{-q^{\prime}} d t\right)^{\frac{1}{q}} \\
& =c_{1}\left(\frac{1}{n q^{\prime}}\right)^{\frac{1}{q}}\left(\sum_{k=k_{0}}^{\infty} \int_{C N_{k+1}^{-n}}^{C N_{k}^{-n}}\left(\frac{f^{*}(t)}{\Phi_{q^{\prime}}(t)^{q^{\prime}}}\right)^{q} t^{-\frac{q^{\prime}}{p}-1} \Lambda\left(t^{-\frac{1}{n}}\right)^{-q^{\prime}} d t\right)^{\frac{1}{q}} \\
& \leqslant c_{2}\left(\sum_{k=k_{0}}^{\infty}\left(\frac{f^{* *}\left(C N_{k+1}^{-n}\right)}{\Phi_{q^{\prime}}\left(C N_{k}^{-n}\right)^{q^{\prime}}}\right)^{q} \sigma_{k}^{-q^{\prime}} N_{k}^{n\left(\frac{q^{\prime}}{p}+1\right)} N_{k}^{-n}\right)^{\frac{1}{q}} \text {. }
\end{aligned}
$$

Based on (4.14) we get the following inequalities:

$$
\begin{aligned}
\left(\int_{0}^{\varepsilon}\left(\frac{f^{*}(t)}{\Phi_{q^{\prime}}(t)}\right)^{q} \mu_{q^{\prime}}(d t)\right)^{\frac{1}{q}} & \\
\leqslant & c_{2}\left(\sum_{k=k_{0}}^{\infty}\left(\frac{\sum_{j=0}^{k} f_{j}^{* *}\left(C N_{k+1}^{-n}\right)}{\Phi_{q^{\prime}}\left(C N_{k}^{-n}\right)^{q^{\prime}}}\right)^{q} \sigma_{k}^{-q^{\prime}} N_{k}^{\frac{n}{p} q^{\prime}}\right)^{\frac{1}{q}} \\
& +c_{2}\left(\sum_{k=k_{0}}^{\infty}\left(\frac{\sum_{j=k+1}^{\infty} f_{j}^{* *}\left(C N_{k+1}^{-n}\right)}{\Phi_{q^{\prime}}\left(C N_{k}^{-n}\right)^{q^{\prime}}}\right)^{q} \sigma_{k}^{-q^{\prime}} N_{k}^{\frac{n}{p} q^{\prime}}\right)^{\frac{1}{q}} \\
= & : S_{1}+S_{2} .
\end{aligned}
$$

We will estimate $S_{1}$ and $S_{2}$ separately. For $0 \leqslant j \leqslant k$ we have $C N_{k+1}^{-n} \in$ $\left(0, C N_{j}^{-n}\right)$. So (3.13), Lemma 2.6, Proposition 2.7, Remark 2.8, Proposition 3.15 and a generalization of Hardy's inequality (cf. [18, p. 247]) allow us to write

$$
\begin{aligned}
S_{1} & \leqslant c_{3}\left(\sum_{k=k_{0}}^{\infty} \sigma_{k}^{-q^{\prime}} N_{k}^{\frac{n}{p} q^{\prime}}\left(\frac{\sum_{j=0}^{k} \sigma_{j}^{-1} N_{j}^{\frac{n}{p}} \lambda_{j, 1}^{*}}{\sum_{j=0}^{k} \sigma_{j}^{-q^{\prime}} N_{j}^{\frac{n}{p} q^{\prime}}}\right)^{q}\right)^{\frac{1}{q}} \\
& \leqslant c_{4}\left\|\left(\lambda_{j m}\right)_{j \in \mathbb{N}_{0}, m \in \mathbb{Z}^{n}} \mid b_{p, q}\right\| .
\end{aligned}
$$


We are going now to estimate $S_{2}$ in (4.15). For $j \geqslant k+1$ we have again $C N_{k+1}^{-n} \in\left[C N_{j}^{-n}\left[N_{j}^{n} N_{k+1}^{-n}\right], C N_{j}^{-n}\left(\left[N_{j}^{n} N_{k+1}^{-n}\right]+1\right)\right)$ and

$$
\lambda_{j,\left[N_{j}^{n} N_{k+1}^{-n}\right]+1}^{* *} \leqslant c_{5} N_{j}^{-\frac{n}{p}} N_{k}^{\frac{n}{p}}\left(\sum_{l=1}^{\infty} \lambda_{j l}^{* * p}\right)^{\frac{1}{p}}
$$

so that from (3.13), Lemma 2.6, Proposition 2.7, Remark 2.8, Proposition 3.15 and a generalized Minkowski inequality we can write

$$
\begin{aligned}
S_{2} & \leqslant c_{6}\left(\sum_{k=k_{0}}^{\infty} \sigma_{k}^{-q^{\prime}} N_{k}^{\frac{n}{p} q^{\prime}}\left(\frac{\sum_{j=k+1}^{\infty} \sigma_{j}^{-1} N_{j}^{\frac{n}{p}} \lambda_{j,\left[N_{j}^{n} N_{k+1}^{-n}\right]+1}^{*}}{\sum_{j=0}^{k} \sigma_{j}^{-q^{\prime}} N_{j}^{\frac{n}{p} q^{\prime}}}\right)^{q}\right)^{\frac{1}{q}} \\
& \leqslant c_{7}\left(\sum_{l=1}^{\infty} \underline{\sigma}_{l}^{-1}\right)\left\|\left(\lambda_{j m}\right)_{j \in \mathbb{N}_{0}, m \in \mathbb{Z}^{n}} \mid b_{p, q}\right\| \\
& \leqslant c_{8}\left\|\left(\lambda_{j m}\right)_{j \in \mathbb{N}_{0}, m \in \mathbb{Z}^{n}} \mid b_{p, q}\right\|
\end{aligned}
$$

due also to our assumption (4.3).

Putting together (4.16), (4.15), and (4.17) we get

$$
\left(\int_{0}^{\varepsilon}\left(\frac{f^{*}(t)}{\Phi_{q^{\prime}}(t)}\right)^{q} \mu_{q^{\prime}}(d t)\right)^{\frac{1}{q}} \leqslant c_{9}\left\|\left(\lambda_{j m}\right)_{j \in \mathbb{N}_{0}, m \in \mathbb{Z}^{n}} \mid b_{p, q}\right\|
$$

for some constant $c_{9}>0$ independent of $f$ and the atomic decomposition taken. Applying the atomic decomposition theorem, Theorem 3.14, the case $1<v=$ $q<\infty$ and $p>1$ of (4.7) follows immediately.

To prove (4.7) for any $v \geqslant q$ (or (4.8) for $v=\infty$ ) one has only to apply [33, Proposition 12.2]. From (4.8) it also easily follows that, still in the case $p>1$, $1<q<\infty$, that (4.6) holds and, in particular, that $\mathcal{E}_{\mathrm{LG}} \mid B_{p, q}^{\sigma, N}(t)$ is finite for each $t \in(0, \varepsilon]$.

Step 3: We assume now that $1<p<\infty$ but $0<q \leqslant 1$ and will prove (4.6) and (4.7).

Again we will start proving (4.7) for $v=q$. Using Lemma 2.6 we have $\Phi_{\infty}\left(C^{-1} t\right) \sim \Phi_{\infty}(t)$ for $t \in\left(0, N_{J_{0}}^{-n}\right]$; in particular this implies $\Phi_{\infty}\left(C N_{k}^{-n}\right) \sim$ $\Phi_{\infty}\left(N_{k}^{-n}\right)$ for any $k \geqslant k_{0}$. Using now Proposition 2.7 for $t=N_{k}^{-n}$ we get

$$
\Phi_{\infty}\left(N_{k}^{-n}\right) \sim \sup _{J_{0} \leqslant j \leqslant k} N_{j}^{\frac{n}{p}} \sigma_{j}^{-1},
$$

so there exist constants $c_{1}>0$ and $c_{2}>0$ such that

$$
c_{1} \sup _{J_{0} \leqslant j \leqslant k} \sigma_{j}^{-1} N_{j}^{\frac{n}{p}} \leqslant \Phi_{\infty}\left(C N_{k}^{-n}\right) \leqslant c_{2} \sup _{J_{0} \leqslant j \leqslant k} \sigma_{j}^{-1} N_{j}^{\frac{n}{p}} \quad \text { for any } k \geqslant k_{0} .
$$


Due to hypothesis (4.4), which in our case is $\left(\sigma_{j}^{-1} N_{j}^{\frac{n}{p}}\right)_{j \in \mathbb{N}_{0}} \notin \ell_{\infty}$, we can construct a strictly increasing sequence $\left(t_{k}\right)_{k \in \mathbb{N}_{0}}$ of natural numbers in the following way:

(i) $t_{0}=k_{0}$, i.e., is such that $C N_{t_{0}}^{-n} \leqslant N_{J_{0}}^{-n}$;

(ii) $t_{k+1}, k \in \mathbb{N}_{0}$, is the smallest integer satisfying

$$
\frac{\sup _{j=J_{0}, \ldots, t_{k+1}} \sigma_{j}^{-1} N_{j}^{\frac{n}{p}}}{\sup _{j=J_{0}, \ldots, t_{k}} \sigma_{j}^{-1} N_{j}^{\frac{n}{p}}} \geqslant \frac{2 c_{2}}{c_{1}}
$$

with $c_{1}, c_{2}$ as in (4.18).

Notice that then

$$
\frac{\sup _{j=J_{0}, \ldots, t_{k}} \sigma_{j}^{-1} N_{j}^{\frac{n}{p}}}{\sup _{j=J_{0}, \ldots, t_{k+1}} \sigma_{j}^{-1} N_{j}^{\frac{n}{p}}} \sim \frac{\sup _{j=J_{0}, \ldots, t_{k}} \sigma_{j}^{-1} N_{j}^{\frac{n}{p}}}{\sup _{j=J_{0}, \ldots, t_{k+1}-1} \sigma_{j}^{-1} N_{j}^{\frac{n}{p}}}>\frac{c_{1}}{2 c_{2}}, \quad k \in \mathbb{N}_{0} .
$$

Denote for any $k \in \mathbb{N}_{0} \alpha_{k}:=C N_{t_{k}}^{-n}$. Then based on (4.18), (4.19), and (4.20), there exists a constant $0<c_{3} \leqslant 1$ such that

$$
c_{3} \frac{1}{2} \leqslant \frac{\Phi_{\infty}\left(\alpha_{k}\right)}{\Phi_{\infty}\left(\alpha_{k+1}\right)} \leqslant \frac{1}{2} \quad \text { for any } k \in \mathbb{N} .
$$

Given $f \in B_{p, q}^{\sigma, N}$ consider a corresponding atomic decomposition $\sum_{j=0}^{\infty} f_{j}$ (convergence in $\mathcal{S}^{\prime}$ ), where $f_{j}$ have the same meaning as in (3.12), for given atoms $a_{j m}$ in $B_{p, q}^{\sigma, N}$ and complex numbers $\lambda_{j m}$ satisfying $\left(\lambda_{j m}\right)_{j \in \mathbb{N}_{0}, m \in \mathbb{Z}^{n}} \in b_{p, q}$. Together with our hypotheses, this guarantees that Proposition 3.17 can be applied with $d_{0}=1, d_{j}:=\sigma_{j}^{-1} N_{j}^{\frac{n}{p}}, j \in \mathbb{N}$. In particular, $f$ is also the limit, in $L_{p}$, of the series $\sum_{j=0}^{\infty} f_{j}$.

Then, recalling also the definition of the measure $\mu_{\infty}$, we have

$$
\begin{aligned}
\left(\int_{0}^{\varepsilon}\left(\frac{f^{*}(t)}{\Phi_{\infty}(t)}\right)^{q} \mu_{\infty}(d t)\right)^{\frac{1}{q}} \leqslant & c_{4}\left(\sum_{k=0}^{\infty} \frac{f^{* *}\left(\alpha_{k+1}\right)^{q}}{\Phi_{\infty}\left(\alpha_{k}\right)^{q}} \mu_{\infty}\left(\left[\alpha_{k+1}, \alpha_{k}\right]\right)\right)^{\frac{1}{q}} \\
\leqslant & c_{5}\left(\sum_{k=0}^{\infty} \frac{1}{\Phi_{\infty}\left(\alpha_{k}\right)^{q}} \sum_{j=0}^{t_{k+1}-1} f_{j}^{* *}\left(\alpha_{k+1}\right)^{q}\right. \\
& \left.+\sum_{k=0}^{\infty} \frac{1}{\Phi_{\infty}\left(\alpha_{k}\right)^{q}} \sum_{j=t_{k+1}}^{\infty} f_{j}^{* *}\left(\alpha_{k+1}\right)^{q}\right)^{\frac{1}{q}} \\
= & : c_{5}\left(S_{1}+S_{2}\right)^{\frac{1}{q}} .
\end{aligned}
$$

We will estimate $S_{1}$ and $S_{2}$ separately. Since for $0 \leqslant j \leqslant t_{k+1}-1$ we have $\alpha_{k+1}=C N_{t_{k+1}}^{-n} \in\left(0, C N_{j}^{-n}\right)$, with the convention $t_{l}=0$ if $l<0$, using (3.13), 
(4.18), (4.21) and the fact $\lambda_{j 1}^{* *}=\lambda_{j 1}^{*}$, we get

$$
\begin{aligned}
S_{1} & \leqslant c_{6} \sum_{k=0}^{\infty} \sum_{h=0}^{k+1}\left(\frac{\Phi_{\infty}\left(\alpha_{h}\right)}{\Phi_{\infty}\left(\alpha_{k}\right)}\right)^{q} \sum_{j=t_{h-1}}^{t_{h}-1} \lambda_{j 1}^{* * q} \\
& \leqslant c_{7}\left(\sum_{l=-1}^{\infty} 2^{-l q}\right)\left(\sum_{j=0}^{\infty} \lambda_{j 1}^{* q}\right) .
\end{aligned}
$$

To estimate $S_{2}$ in (4.22) let now $j \geqslant t_{k+1}$. Then $C N_{t_{k+1}}^{-n} \in\left[C N_{j}^{-n}\left[N_{j}^{n} N_{t_{k+1}}^{-n}\right]\right.$, $\left.C N_{j}^{-n}\left(\left[N_{j}^{n} N_{t_{k+1}}^{-n}\right]+1\right)\right)$ so that, using (3.13),

$$
\sum_{j=t_{k+1}}^{\infty} f_{j}^{* *}\left(\alpha_{k+1}\right)^{q} \leqslant c_{8} \sum_{j=t_{k+1}}^{\infty}\left(\sigma_{j}^{-1} N_{j}^{\frac{n}{p}}\right)^{q} \lambda_{j,\left[N_{j}^{n} N_{t_{k+1}}^{-n}\right]+1}^{* q}
$$

and, as in Step 1,

$$
\lambda_{j,\left[N_{j}^{n} N_{t_{k+1}}^{-n}\right]+1}^{* *} \leqslant c_{9} N_{j}^{-\frac{n}{p}} N_{t_{k+1}}^{\frac{n}{p}}\left(\sum_{l=1}^{\infty} \lambda_{j l}^{* * p}\right)^{\frac{1}{p}} .
$$

Inserting this in (4.24), and using $\Phi_{\infty}\left(\alpha_{k}\right) \geqslant c_{12} \sigma_{t_{k+1}}^{-1} N_{t_{k+1}}^{\frac{n}{p}}$ we get for $S_{2}$, again with the help of Proposition 3.15

$$
\begin{aligned}
S_{2} & \leqslant c_{10} \sum_{k=0}^{\infty} \frac{1}{\Phi_{\infty}\left(\alpha_{k}\right)^{q}} \sum_{j=t_{k+1}}^{\infty} \sigma_{j}^{-q} N_{j}^{\frac{n}{p} q} N_{j}^{-\frac{n}{p} q} N_{t_{k+1}}^{\frac{n}{p} q}\left(\sum_{l=1}^{\infty} \lambda_{j l}^{* * p}\right)^{\frac{q}{p}} \\
& \leqslant c_{11}\left(\sum_{l=0}^{\infty} \underline{\sigma}_{l}^{-q}\right)\left\|\left(\lambda_{j m}\right)_{j \in \mathbb{N}_{0}, m \in \mathbb{Z}^{n}} \mid b_{p, q}\right\|^{q} .
\end{aligned}
$$

Putting (4.23) and (4.25) in (4.22) and using our assumption (4.3) we get

$$
\left(\int_{0}^{\varepsilon}\left(\frac{f^{*}(t)}{\Phi_{\infty}(t)}\right)^{q} \mu_{\infty}(d t)\right)^{\frac{1}{q}} \leqslant c_{12}\left\|\left(\lambda_{j m}\right)_{j \in \mathbb{N}_{0}, m \in \mathbb{Z}^{n}} \mid b_{p, q}\right\|
$$

for some constant $c_{12}>0$ independent of $f$ and the atomic decomposition taken. A simple application of Theorem 3.14 proves (4.7) in the case $0<v=q \leqslant 1$ (and $p>1$ ).

To prove (4.7) for any $v \geqslant q$ (even for $v=\infty$, in which case we are thinking on (4.8)) one has again only to apply [33, Proposition 12.2]. From (4.8) it also easily follows, still in the case $p>1,0<q \leqslant 1$, that (4.6) holds and, in particular, that $\mathcal{E}_{\mathrm{LG}} \mid B_{p, q}^{\sigma, N}(t)$ is finite for each $t \in(0, \varepsilon]$.

Step 4: We will extend now the validity of (4.6) and (4.7) to the case when $0<p \leqslant 1$. There is no loss of generality in assuming $0<\delta<1$ in (4.3). Then 
let $\gamma=\frac{\delta}{n-\delta}>0$ and consider the (clearly) admissible sequence $\beta=\left(\beta_{j}\right)_{j \in \mathbb{N}_{0}}$, where $\beta_{j}=\sigma_{j} N_{j}^{\frac{n}{1+\gamma}} N_{j}^{-\frac{n}{p}}$ for any $j \in \mathbb{N}_{0}$. A simple computation shows that

$$
\left(\sigma_{j}^{-1} \beta_{j} N_{j}^{n\left(\frac{1}{p}-\frac{1}{1+\gamma}\right)}\right)_{j \in \mathbb{N}_{0}} \in \ell_{\infty}
$$

so that based on the embedding result stated in Theorem 3.7 we have

$$
B_{p, q}^{\sigma, N} \hookrightarrow B_{1+\gamma, q}^{\beta, N}
$$

Since

$$
\left(\beta_{j}^{-1} N_{j}^{\frac{1}{1+\gamma}}\right)_{j \in \mathbb{N}_{0}} \notin \ell_{q^{\prime}} \quad \text { and } \quad\left(\underline{\beta}_{j}^{-1}\right)_{j \in \mathbb{N}_{0}} \in \ell_{\min (q, 1)}
$$

we may apply Steps $1-3$ to the space $B_{1+\gamma, q}^{\beta, N}$.

Let $\Gamma$ be an admissible function such that $\Gamma(z) \sim \beta_{j}, z \in\left[N_{j}, N_{j+1}\right], j \in \mathbb{N}_{0}$, with equivalence constants independent of $j$, and let $\Psi_{q^{\prime}}$, defined in $\left(0, N_{J_{0}}^{-n}\right]$, be the function from Definition 2.4 (with $q^{\prime}$ instead of $u, 1+\gamma$ instead of $p$ and $\Gamma$ instead of $\Lambda$ ), where $J_{0} \in \mathbb{N}$ is chosen such that $N_{J_{0}}>1$. Let $\theta_{q^{\prime}}$ be the Borel measure associated with $-\log _{2} \Psi_{q^{\prime}}$. From the previous steps we have

$$
\left(\int_{0}^{\varepsilon}\left(\frac{f^{*}(t)}{\Psi_{q^{\prime}}(t)}\right)^{v} \theta_{q^{\prime}}(d t)\right)^{\frac{1}{v}} \leqslant c(v)\left\|f \mid B_{1+\gamma, q}^{\beta, N}\right\| \quad \text { for any } f \in B_{1+\gamma, q}^{\beta, N},
$$

with appropriate change if $v=\infty$. Using Proposition 2.7 we have (appropriate modification if $q^{\prime}=\infty$ )

$$
\Psi_{q^{\prime}}(t) \sim\left(\sum_{j=J_{0}}^{k}\left(\beta_{j}^{-1} N_{j}^{\frac{n}{1+\gamma}}\right)^{q^{\prime}}\right)^{\frac{1^{\prime}}{q}} \sim\left(\sum_{j=J_{0}}^{k}\left(\sigma_{j}^{-1} N_{j}^{\frac{n}{p}}\right)^{q^{\prime}}\right)^{\frac{1^{\prime}}{q}} \sim \Phi_{q^{\prime}}(t)
$$

for $t \in\left(0, N_{J_{0}}^{-n}\right]$, so that combining this with (4.26), (4.27) and [33, Proposition 12.2] we get (4.7) for $v<\infty$, or (4.8) if $v=\infty$. Similarly as in the previous steps one gets also (4.6) and the finiteness of $\mathcal{E}_{\mathrm{LG}} \mid B_{p, q}^{\sigma, N}(t)$ for $t \in(0, \varepsilon]$.

4.3. Estimates from below. The following lemma is essential in this section, but might be also of independent interest.

Lemma 4.6. Given $L \in \mathbb{N}$ and $\lambda_{0}>1$, there exists a $C^{\infty}$-function $\phi$ in $\mathbb{R}^{n}$ for which

(i) there are positive constants $C_{1}, C_{2}$ and $C_{3}$ with $C_{1}<C_{3}<\lambda_{0} C_{1}$ such that

$$
\begin{array}{ll}
\phi(x) \geqslant C_{2} & \text { whenever }|x|_{\infty} \leqslant C_{1} \\
\phi(x)=0 & \text { whenever }|x|_{\infty} \geqslant C_{3}
\end{array}
$$


(ii) $\int_{\mathbb{R}^{n}} x^{\gamma} \phi(x) d x=0$ whenever $\gamma_{1}+\ldots+\gamma_{n} \leqslant L$ with $\gamma=\left(\gamma_{j}\right)_{j=1}^{n} \in \mathbb{N}_{0}^{n}$.

Moreover, $C_{1}, C_{2}$ and $C_{3}$ can be chosen to depend only on $n$ and $\lambda_{0}$.

Proof. Consider $h(y):=e^{-\frac{1}{1-y^{2}}}$ if $|y|<1$ and $h(y):=0$ if $|y| \geqslant 1$. Given $L \in \mathbb{N}$ and $\delta \in(0,1]$, define

$$
h_{\delta, L}(y):=h(y)-\sum_{l=0}^{L} \rho_{\delta, L} h^{(l)}\left(\delta^{-1}(y-1-\delta)\right),
$$

where the coefficients $\rho_{\delta, L}$ are uniquely determined by imposing that $h_{\delta, L}$ obey the following set of conditions:

$$
\int_{\mathbb{R}} y^{k} h_{\delta, L}(y) d y=0, \quad k=0, \ldots, L .
$$

In fact, this follows easily by plugging (4.28) in (4.29) and noticing that the system obtained has triangular matrix with nonzero determinant. Let now

$$
\phi_{\delta, L}(x):=h_{\delta, L}\left(x_{1}\right) \prod_{m=2}^{n} h\left(x_{m}\right), \quad x:=\left(x_{j}\right)_{j=1}^{n} \in \mathbb{R}^{n} .
$$

By using (4.29) it is easily verified that $\phi_{\delta, L}$ obeys the required moment conditions until the order $L$ (that is, condition (ii) above, with $\phi_{\delta, L}$ instead of $\phi$ ).

On the other hand, it is also easy to see that

$$
\phi_{\delta, L}(x)=0 \text { if }|x|_{\infty} \geqslant 1+2 \delta \text { and } \quad \phi_{\delta, L}(x) \geqslant e^{-\frac{n}{2 \delta-\delta^{2}}} \text { if }|x|_{\infty} \leqslant 1-\delta,
$$

so that condition (i) above is satisfied for $C_{1}:=1-\delta, C_{2}:=e^{-\frac{n}{2 \delta-\delta^{2}}}, C_{3}:=1+2 \delta$ and $\phi:=\phi_{\delta, L}$ if $\delta$ is chosen in $\left(0, \frac{\lambda_{0}-1}{2+\lambda_{0}}\right)$, which is clearly possible because $\lambda_{0}>1$. The proof is complete.

Proposition 4.7. Let $N:=\left(N_{j}\right)_{j \in \mathbb{N}_{0}}$ satisfy Assumption 2 and $\sigma=\left(\sigma_{j}\right)_{j \in \mathbb{N}_{0}}$ be an admissible sequence (with equivalence constants $\kappa_{0}, \kappa_{1}$ ). Let $0<p, q \leqslant \infty$. Let $b:=\left(b_{j}\right)_{j \in \mathbb{N}} \subset \mathbb{C}$ be an eventually null sequence (that is, for which there exists $T \in \mathbb{N}$ such that $b_{j}=0$ for $j \geqslant T$ ) and $L \in \mathbb{N}$ satisfy (3.10). Then the function $f^{b}$ given by

$$
f^{b}(x):=\sum_{j=1}^{\infty} b_{j} \sigma_{j}^{-1} N_{j}^{\frac{n}{p}} \phi\left(N_{j} x\right), \quad x \in \mathbb{R}^{n},
$$

where $\phi$ is a function fixed in accordance with the previous lemma, for the $\lambda_{0}$ and $L$ considered now, belongs to $B_{p, q}^{\sigma, N}$ and there exists $c_{1}>0$ (independent of b) such that

$$
\left\|f^{b}\left|B_{p, q}^{\sigma, N}\left\|\leqslant c_{1}\right\| b\right| \ell_{q}\right\|
$$


If, moreover, $b_{j} \geqslant 0$, for all $j \in \mathbb{N}$, then there also exist $c_{2} \in(0,1]$ and $c_{3}>0$ (independent of $b$ and $k$ ) such that

$$
\left(f^{b}\right)^{*}\left(c_{2} N_{k}^{-n}\right) \geqslant c_{3} \sum_{j=1}^{k} b_{j} \sigma_{j}^{-1} N_{j}^{\frac{n}{p}}, \quad k \in \mathbb{N} .
$$

Proof. Since the functions $a_{j}(x):=\sigma_{j}^{-1} N_{j}^{\frac{n}{p}} \phi\left(N_{j} x\right), x \in \mathbb{R}^{n}, j \in \mathbb{N}$, are (up to constants, independently of $j)(\sigma, p)_{M, L^{-}} N$-atoms, for some fixed $M \in \mathbb{N}$ with $M>\frac{\log _{2} \kappa_{1}}{\log _{2} \lambda_{0}}$, and $b \in \ell_{q}$, then (4.31) is an immediate consequence of Theorem 3.14.

Consider now that $b_{j} \geqslant 0$ for all $j \in \mathbb{N}$. Let $C_{1}, C_{2}$ and $C_{3}$ be the constants associated with the fixed $\phi$, as in Lemma 4.6. Note that whenever $x \in \mathbb{R}^{n}$ and $k \in \mathbb{N}$ satisfy the relation $C_{3} \lambda_{0}^{-1} N_{k}^{-1} \leqslant|x|_{\infty} \leqslant C_{1} N_{k}^{-1}$, it holds $\phi\left(N_{j} x\right) \geqslant C_{2}$ if $1 \leqslant j \leqslant k$ and $\phi\left(N_{j} x\right)=0$ if $j>k$, and therefore

$$
f^{b}(x) \geqslant C_{2} \sum_{j=1}^{k} b_{j} \sigma_{j}^{-1} N_{j}^{\frac{n}{p}}
$$

Let now $k \in \mathbb{N}$ be given and $0 \leqslant \lambda<C_{2} \sum_{j=1}^{k} b_{j} \sigma_{j}^{-1} N_{j}^{\frac{n}{p}}$. Then

$$
\begin{aligned}
m_{f^{b}}(\lambda) & \geqslant\left|\left\{x \in \mathbb{R}^{n}:\left|f^{b}(x)\right| \geqslant C_{2} \sum_{j=1}^{k} b_{j} \sigma_{j}^{-1} N_{j}^{\frac{n}{p}}\right\}\right| \\
& \geqslant\left|\left\{x \in \mathbb{R}^{n}: C_{3} \lambda_{0}^{-1} N_{k}^{-1} \leqslant|x|_{\infty} \leqslant C_{1} N_{k}^{-1}\right\}\right| \\
& =2^{n}\left(C_{1}^{n}-C_{3}^{n} \lambda_{0}^{-n}\right) N_{k}^{-n},
\end{aligned}
$$

hence for $0 \leqslant t<2^{n}\left(C_{1}^{n}-C_{3}^{n} \lambda_{0}^{-n}\right) N_{k}^{-n}$ one has $\left(f^{b}\right)^{*}(t) \geqslant C_{2} \sum_{j=1}^{k} b_{j} \sigma_{j}^{-1} N_{j}^{\frac{n}{p}}$, so the proof is complete if one chooses $c_{2}:=\min \left\{1,2^{n-1}\left(C_{1}^{n}-C_{3}^{n} \lambda_{0}^{-n}\right)\right\}$ and $c_{3}:=C_{2}$.

Proposition 4.8. Let $0<p, q \leqslant \infty$. Let $N:=\left(N_{j}\right)_{j \in \mathbb{N}_{0}}$ satisfy Assumption 2 and $\sigma:=\left(\sigma_{j}\right)_{j \in \mathbb{N}_{0}}$ be an admissible sequence. Assume that $B_{p, q}^{\sigma, N} \subset L_{1}^{\text {loc }}$. Then there exists $c>0$ such that

$$
\mathcal{E}_{L G} \mid B_{p, q}^{\sigma, N}(t) \geqslant c \Phi_{q^{\prime}}(t), \quad t \in\left(0, c_{2} N_{J_{0}}^{-n}\right],
$$

where $c_{2} \in(0,1]$ is the constant with the same name in Proposition $4.7, J_{0} \in \mathbb{N}$ is chosen such that $N_{J_{0}}>1$ and $\Phi_{q^{\prime}}$, defined in $\left(0, N_{J_{0}}^{-n}\right]$, is the function of Definition 2.4 (with $q^{\prime}$ instead of $u$ ) built by means of an admissible function $\Lambda$ such that $\Lambda(z) \sim \sigma_{j}$, for $z \in\left[N_{j}, N_{j+1}\right], j \in \mathbb{N}_{0}$, with equivalence constants independent of $j$. 
Proof. Step 1: Let $1<q \leqslant \infty$. For each $J \in \mathbb{N}$, denote by $f_{J}$ the function $f^{b}$ in (4.30) with $b:=\left(b_{j}\right)_{j \in \mathbb{N}}$ the sequence defined by

$$
b_{j}:= \begin{cases}\sigma_{j}^{1-q^{\prime}} N_{j}^{-\frac{n}{p}\left(1-q^{\prime}\right)}\left(\sum_{k=1}^{J} \sigma_{k}^{-q^{\prime}} N_{k}^{\frac{n}{p} q^{\prime}}\right)^{-\frac{1}{q}} & \text { for } j=1, \ldots, J, \\ 0 & \text { otherwise. }\end{cases}
$$

Notice that $\left\|b \mid \ell_{q}\right\|=1$. By (4.32), and using the same constants,

$$
f_{J}^{*}\left(c_{2} N_{J}^{-n}\right) \geqslant c_{3}\left(\sum_{j=1}^{J} \sigma_{j}^{-q^{\prime}} N_{j}^{\frac{n}{p} q^{\prime}}\right)^{\frac{1}{q^{\prime}}}
$$

and by (4.31) and the property $(\lambda f)^{*}=|\lambda| f^{*}$ we obtain

$$
\mathcal{E}_{\mathrm{LG}} \mid B_{p, q}^{\sigma, N}\left(c_{2} N_{J}^{-n}\right) \geqslant c_{1}^{-1} c_{3}\left(\sum_{j=1}^{J} \sigma_{j}^{-q^{\prime}} N_{j}^{\frac{n}{p} q^{\prime}}\right)^{\frac{1}{q^{\prime}}}, \quad J \in \mathbb{N} .
$$

Now let, for each given $t \in\left(0, c_{2} N_{J_{0}}^{-n}\right], J \geqslant J_{0}$ be such that $c_{2} N_{J+1}^{-n}<$ $t \leqslant c_{2} N_{J}^{-n}$. Using (4.33), the monotonicity of $\mathcal{E}_{\mathrm{LG}} \mid B_{p, q}^{\sigma, N}$, our hypotheses on $\Lambda$, Proposition 2.7 and Lemma 2.6, we finally get

$$
\mathcal{E}_{\mathrm{LG}} \mid B_{p, q}^{\sigma, N}(t) \geqslant c_{4}\left(\sum_{j=J_{0}}^{J} \Lambda\left(N_{j}\right)^{-q^{\prime}} N_{j}^{\frac{n}{p} q^{\prime}}\right)^{\frac{1}{q^{\prime}}} \geqslant c_{5} \Phi_{q^{\prime}}\left(c_{2}^{-1} t\right) \geqslant c_{6} \Phi_{q^{\prime}}(t) .
$$

Step 2: Case $0<q \leqslant 1$. For each $k \in \mathbb{N}$, denote by $f_{k}$ the function $f^{b}$ in (4.30) with $b:=\left(b_{j}\right)_{j \in \mathbb{N}}$ the sequence defined by

$$
b_{j}:= \begin{cases}1 & \text { for } j=k \\ 0 & \text { otherwise }\end{cases}
$$

Notice that $\left\|b \mid \ell_{q}\right\|=1$. By (4.32), and using the same constants,

$$
f_{k}^{*}\left(c_{2} N_{J}^{-n}\right) \geqslant c_{3} \sigma_{k}^{-1} N_{k}^{\frac{n}{p}} \quad \text { whenever } \quad J \geqslant k(k, J \in \mathbb{N}) .
$$

Now let, for each given $t \in\left(0, c_{2} N_{J_{0}}^{-n}\right], J \geqslant J_{0}$ be such that $c_{2} N_{J+1}^{-n}<t \leqslant$ $c_{2} N_{J}^{-n}$. Using (4.34), the monotonicity of $\mathcal{E}_{\mathrm{LG}} \mid B_{p, q}^{\sigma, N},(4.31)$, the property $(\lambda f)^{*}=$ $|\lambda| f^{*}$, our hypotheses on $\Lambda$, Proposition 2.7 and Lemma 2.6, we finally get

$$
\begin{aligned}
\mathcal{E}_{\mathrm{LG}} \mid B_{p, q}^{\sigma, N}(t) & \geqslant \sup \left\{f^{*}\left(c_{2} N_{J}^{-n}\right):\left\|f \mid B_{p, q}^{\sigma, N}\right\| \leqslant 1\right\} \\
& \geqslant c_{1}^{-1} \sup \left\{f_{k}^{*}\left(c_{2} N_{J}^{-n}\right): k=J_{0}, \ldots, J\right\} \\
& \geqslant c_{4} \sup \left\{\Lambda\left(N_{k}\right)^{-1} N_{k}^{\frac{n}{p}}: k=J_{0}, \ldots, J\right\} \\
& \geqslant c_{5} \Phi_{\infty}\left(c_{2}^{-1} t\right) \\
& \geqslant c_{6} \Phi_{\infty}(t) .
\end{aligned}
$$


We are now ready to prove the converse to Corollary 3.10.

Corollary 4.9. Let $N=\left(N_{j}\right)_{j \in \mathbb{N}_{0}}$ be an admissible sequence with $\lambda_{0}>1$ in (3.1) and let $\sigma=\left(\sigma_{j}\right)_{j \in \mathbb{N}_{0}}$ be an admissible sequence. Let $0<p \leqslant \infty$ and $0<q \leqslant \infty$. If $B_{p, q}^{\sigma, N} \hookrightarrow C$, then $\left(\sigma_{j}^{-1} N_{j}^{\frac{n}{p}}\right)_{j \in \mathbb{N}_{0}} \in \ell_{q^{\prime}}$.

Proof. Since the assumption $B_{p, q}^{\sigma, N} \hookrightarrow C$ implies that $B_{p, q}^{\sigma, N} \subset L_{1}^{\text {loc }}$, we can use the proof of the preceding proposition and state that

$$
\mathcal{E}_{\mathrm{LG}} \mid B_{p, q}^{\sigma, N}\left(c_{2} N_{J}^{-n}\right) \geqslant c_{1}^{-1} c_{3}\left(\sum_{j=1}^{J} \sigma_{j}^{-q^{\prime}} N_{j}^{\frac{n}{p} q^{\prime}}\right)^{\frac{1}{q^{\prime}}}, \quad J \in \mathbb{N}
$$

(usual modification if $q^{\prime}=\infty$ ), for some positive constants $c_{1}, c_{2}$ and $c_{3}$ as before. On the other hand, $B_{p, q}^{\sigma, N} \hookrightarrow C \hookrightarrow L_{\infty}$ imply that for any $f \in B_{p, q}^{\sigma, N}$ with $\left\|f\left|B_{p, q}^{\sigma, N}\left\|\leqslant 1, f^{*}(0)=\right\| f\right| L_{\infty}\right\| \leqslant c_{4}$, for some constant $c_{4}>0$, and therefore

$$
\mathcal{E}_{\mathrm{LG}} \mid B_{p, q}^{\sigma, N}(t) \leqslant c_{4}, \quad t>0 .
$$

The result follows from the last inequality and from (4.35).

\subsection{The main theorem; examples and remarks.}

Theorem 4.10. Let $0<p, q \leqslant \infty$. Let $N=\left(N_{j}\right)_{j \in \mathbb{N}_{0}}$ satisfy Assumption 2 and $\sigma:=\left(\sigma_{j}\right)_{j \in \mathbb{N}_{0}}$ be an admissible sequence. Assume that (4.3) and (4.4) both hold. Let $\Lambda$ be an admissible function such that $\Lambda(z) \sim \sigma_{j}, z \in\left[N_{j}, N_{j+1}\right], j \in \mathbb{N}_{0}$, with equivalence constants independent of $j$, and let $\Phi_{q^{\prime}}$, defined in $\left(0, N_{J_{0}}^{-n}\right]$, be the function from Definition 2.4 (with $q^{\prime}$ instead of $u$ ), where $J_{0} \in \mathbb{N}$ is chosen such that $N_{J_{0}}>1$. Then

$$
\mathfrak{E}_{\mathrm{LG}} B_{p, q}^{\sigma, N}=\left(\Phi_{q^{\prime}}, q\right)
$$

Proof. We remark that, in view of Propositions 4.5 and 4.8, we have just to prove the optimality of the exponent $q$.

Step 1: Let first $1<q \leqslant \infty$. Assume that for some $v \in(0, \infty)$ there is a constant $c(v)>0$ such that

$$
\left(\int_{0}^{\varepsilon}\left(\frac{f^{*}(t)}{\Phi_{q^{\prime}}(t)}\right)^{v} \mu_{q^{\prime}}(d t)\right)^{\frac{1}{v}} \leqslant c(v)\left\|f \mid B_{p, q}^{\sigma, N}\right\|, \quad \text { for all } f \in B_{p, q}^{\sigma, N},
$$

where $\mu_{q^{\prime}}$ denotes the Borel measure associated with $-\log _{2} \Phi_{q^{\prime}}$ in $(0, \varepsilon]$, for some $0<\varepsilon \leqslant N_{J_{0}}^{-n}$. Notice that, by Proposition 2.7 and Lemma 2.6, there are positive constants $c_{7}, c_{8}$ such that

$$
c_{7}\left(\sum_{j=J_{0}}^{k}\left(\sigma_{j}^{-1} N_{j}^{\frac{n}{p}}\right)^{q^{\prime}}\right)^{\frac{1}{q^{\prime}}} \leqslant \Phi_{q^{\prime}}\left(c_{2} N_{k}^{-n}\right) \leqslant c_{8}\left(\sum_{j=J_{0}}^{k}\left(\sigma_{j}^{-1} N_{j}^{\frac{n}{p}}\right)^{q^{\prime}}\right)^{\frac{1}{q^{\prime}}}, \quad k \geqslant J_{0},
$$


where $c_{2} \in(0,1]$ has the same meaning as in Proposition 4.7 .

Due to hypothesis (4.4) we can construct a strictly increasing sequence $\left(t_{k}\right)_{k \in \mathbb{N}_{0}}$ of natural numbers in the following way:

(i) $t_{0}$ is such that $N_{t_{0}}^{-n} \leqslant \varepsilon$;

(ii) $t_{k+1}, k \in \mathbb{N}_{0}$, is the smallest integer satisfying

$$
\frac{\sum_{j=J_{0}}^{t_{k+1}}\left(\sigma_{j}^{-1} N_{j}^{\frac{n}{p}}\right)^{q^{\prime}}}{\sum_{j=J_{0}}^{t_{k}}\left(\sigma_{j}^{-1} N_{j}^{\frac{n}{p}}\right)^{q^{\prime}}} \geqslant\left(\frac{2 c_{8}}{c_{7}}\right)^{q^{\prime}}
$$

with $c_{7}, c_{8}$ as in (4.38).

We remark that in such a case, for all $k \in \mathbb{N}_{0}$,

$$
\frac{\sum_{j=J_{0}}^{t_{k+1}}\left(\sigma_{j}^{-1} N_{j}^{\frac{n}{p}}\right)^{q^{\prime}}}{\sum_{j=J_{0}}^{t_{k}}\left(\sigma_{j}^{-1} N_{j}^{\frac{n}{p}}\right)^{q^{\prime}}} \sim 1
$$

For each $J \in \mathbb{N}$, let $b=\left(b_{j}\right)_{j \in \mathbb{N}}$ be defined by

$$
b_{j}:= \begin{cases}\left(\sigma_{j}^{-1} N_{j}^{\frac{n}{p}}\right)^{-1+q^{\prime}}\left(\sum_{l=J_{0}}^{t_{k}}\left(\sigma_{l}^{-1} N_{l}^{\frac{n}{p}}\right)^{q^{\prime}}\right)^{-\frac{1}{q}} & \text { for } \begin{array}{c}
j=t_{k-1}+1, \ldots, t_{k} \\
k=1, \ldots, J
\end{array} \\
0 & \text { otherwise } .\end{cases}
$$

We have

$$
\left\|b \mid \ell_{q}\right\|=\left(\sum_{k=1}^{J} \sum_{j=t_{k-1}+1}^{t_{k}}\left(\sigma_{j}^{-1} N_{j}^{\frac{n}{p}}\right)^{q^{\prime}}\left(\sum_{l=J_{0}}^{t_{k}}\left(\sigma_{l}^{-1} N_{l}^{\frac{n}{p}}\right)^{q^{\prime}}\right)^{-1}\right)^{\frac{1}{q}} \leqslant J^{\frac{1}{q}}
$$

(with the usual modification if $q=\infty$ ). Clearly, $b_{j} \geqslant 0$, for all $j \in \mathbb{N}$. Denote by $f_{J}$ the corresponding function $f^{b}$ of Proposition 4.7. Then

$$
\left\|f_{J} \mid B_{p, q}^{\sigma, N}\right\| \leqslant c_{1} J^{\frac{1}{q}}
$$

and

$$
\begin{aligned}
f_{J}^{*}\left(c_{2} N_{t_{k}}^{-n}\right) & \geqslant c_{3} \sum_{j=t_{k-1}+1}^{t_{k}} b_{j} \sigma_{j}^{-1} N_{j}^{\frac{n}{p}} \\
& =c_{3}\left(\sum_{l=J_{0}}^{t_{k}}\left(\sigma_{l}^{-1} N_{l}^{\frac{n}{p}}\right)^{q^{\prime}}\right)^{-\frac{1}{q}} \sum_{j=t_{k-1}+1}^{t_{k}}\left(\sigma_{j}^{-1} N_{j}^{\frac{n}{p}}\right)^{q^{\prime}}, \quad k \in\{1, \ldots, J\},
\end{aligned}
$$


From (4.37), using (4.41), the monotonicity of $f_{J}^{*}$ and $\Phi_{q^{\prime}},(4.42),(4.38),(4.39)$ and (4.40), we obtain for any $J \in \mathbb{N}$,

$$
\begin{aligned}
J^{\frac{1}{q}} & \geqslant c\left(\sum_{k=1}^{J}\left(\frac{f_{J}^{*}\left(c_{2} N_{t_{k}}^{-n}\right)}{\Phi_{q^{\prime}}\left(c_{2} N_{t_{k+1}}^{-n}\right)}\right)^{v} \mu_{q^{\prime}}\left(\left[c_{2} N_{t_{k+1}}^{-n}, c_{2} N_{t_{k}}^{-n}\right]\right)\right)^{\frac{1}{v}} \\
& \geqslant c^{\prime}\left\{\sum_{k=1}^{J}\left(\sum_{j=t_{k-1}+1}^{t_{k}}\left(\sigma_{j}^{-1} N_{j}^{\frac{n}{p}}\right)^{q^{\prime}}\right)^{v}\left(\sum_{j=J_{0}}^{t_{k}}\left(\sigma_{j}^{-1} N_{j}^{\frac{n}{p}}\right)^{q^{\prime}}\right)^{-v}\right\}^{\frac{1}{v}} \\
& \geqslant c^{\prime \prime} J^{\frac{1}{v}}
\end{aligned}
$$

This being true for all $J \in \mathbb{N}$, then it must be $v \geqslant q$.

Step 2: Now let $0<q \leqslant 1$. Then $q^{\prime}=\infty$. We modify appropriately Step 1 . Assume that $(4.37)$ holds true for some $v \in(0, \infty)$. The counterpart of $(4.38)$ reads as follows:

$$
c_{7} \sup _{j=J_{0}, \ldots, k} \sigma_{j}^{-1} N_{j}^{\frac{n}{p}} \leqslant \Phi_{\infty}\left(c_{2} N_{k}^{-n}\right) \leqslant c_{8} \sup _{j=J_{0}, \ldots, k} \sigma_{j}^{-1} N_{j}^{\frac{n}{p}}, \quad k \geqslant J_{0} .
$$

Due to hypothesis (4.4), we can construct a strictly increasing sequence $\left(t_{k}\right)_{k \in \mathbb{N}_{0}}$ of natural numbers as in the preceding step, now with supremums instead of sums (cf. also the construction in Step 3 of the proof of Proposition 4.5).

For each $J \in \mathbb{N}$, let $b=\left(b_{j}\right)_{j \in \mathbb{N}}$ be defined by

$$
b_{j}:= \begin{cases}1 & \text { if } j=t_{k}, k \in\{1, \cdots, J\} \\ 0 & \text { otherwise. }\end{cases}
$$

We have $\left\|b \mid \ell_{q}\right\|=J^{\frac{1}{q}}$. Then the corresponding function $f_{J}$, as in Step 1, satisfies

$$
\left\|f_{J} \mid B_{p, q}^{\sigma, N}\right\| \leqslant c_{1} J^{\frac{1}{q}} \quad \text { and } \quad f_{J}^{*}\left(c_{2} N_{t_{k}}^{-n}\right) \geqslant c_{3} \sigma_{t_{k}}^{-1} N_{t_{k}}^{\frac{n}{p}}, \quad k \in\{1, \ldots J\},
$$

where $c_{1}, c_{2}$ and $c_{3}$ are as in Proposition 4.7. From (4.37), using the monotonicity of $f_{J}^{*}$ and $\Phi_{\infty},(4.43),(4.44)$ and the properties of the sequence $\left(t_{k}\right)_{k \in \mathbb{N}_{0}}$, we obtain for any $J \in \mathbb{N}$,

$$
\begin{aligned}
J^{\frac{1}{q}} & \geqslant c\left(\sum_{k=1}^{J}\left(\frac{f_{J}^{*}\left(c_{2} N_{t_{k}}^{-n}\right)}{\Phi_{\infty}\left(c_{2} N_{t_{k+1}}^{-n}\right)}\right)^{v} \mu_{\infty}\left(\left[c_{2} N_{t_{k+1}}^{-n}, c_{2} N_{t_{k}}^{-n}\right]\right)\right)^{\frac{1}{v}} \\
& \geqslant c^{\prime}\left\{\sum_{k=1}^{J}\left(\frac{\sigma_{t_{k}}^{-1} N_{t_{k}}^{\frac{n}{p}}}{\sup _{j=J_{0}, \ldots, t_{k+1}} \sigma_{j}^{-1} N_{j}^{\frac{n}{p}}}\right)^{v}\right\}^{\frac{1}{v}} \\
& \geqslant c^{\prime \prime} J^{\frac{1}{v}}
\end{aligned}
$$

This being true for all $J \in \mathbb{N}$, then it must be $v \geqslant q$. 
Remark 4.11. Observe that the hypothesis (4.3) was not needed in Steps 1 and 2 of the preceding proof, but merely that $B_{p, q}^{\sigma, N} \subset L_{1}^{\text {loc }}$. For the effect of what is proved in Steps 1 and 2, we can even drop this assumption, since what is actually proved there is that a property like (4.37) cannot stand for all regular (even smooth) members of $B_{p, q}^{\sigma, N}$ if $v$ is less than $q$ (as long as we keep all the other hypotheses, of course). Don't forget, however, that Steps 1 and 2 deal only with part of the proof of formula (4.36).

Remark 4.12. We would like to point out that the theorem covers and extends the results previously obtained by A. Caetano and S. Moura [4], [5], which already covered the general statements of D. Haroske [19] and H. Triebel [33], as far as growth envelopes for Besov-type spaces are concerned. To see this, one just has to show that the spaces considered in Theorem 4.4 of [5] are included in our main theorem. It is, in fact, an easy exercise to show that the spaces $B_{p, q}^{\sigma, N}$ considered there, namely with $N_{j}=2^{j}$ and $\sigma_{j}=2^{j s} \Psi\left(2^{-j}\right), j \in \mathbb{N}_{0}$, where $n\left(\frac{1}{p}-1\right)_{+}<s \leqslant \frac{n}{p}$ and $\Psi$ is a so-called admissible function (in the context of that paper) satisfying $\left(\Psi\left(2^{-j}\right)^{-1}\right)_{j \in \mathbb{N}} \notin \ell_{q^{\prime}}$ when $s=\frac{n}{p}$, are such that $\left(\sigma_{j}\right)_{j \in \mathbb{N}_{0}}$ is admissible (in our sense), $\left(N_{j}\right)_{j \in \mathbb{N}_{0}}$ satisfies Assumption 2 and, moreover, (4.3) and (4.4) hold.

Remark 4.13. Our main theorem also covers and extends Theorem 6.3 (i) of [2], where it is again an easy exercise to see that the spaces $B_{p, q}^{\sigma, N}$ considered there, namely with $N_{j}=2^{j}, j \in \mathbb{N}_{0}$, and $\sigma$ an admissible sequence satisfying

$$
n\left(\frac{1}{p}-1\right)_{+}<\lim _{l \rightarrow \infty} \frac{\log _{2} \underline{\sigma}_{l}}{l} \leqslant \lim _{l \rightarrow \infty} \frac{\log _{2} \bar{\sigma}_{l}}{l}<\frac{n}{p},
$$

verify all the requirements of our main theorem.

In particular, the representatives for the corresponding growth envelope functions, though possibly different, must all be equivalent near 0 , for each fixed set of parameters. The situation is similar to what already happened when comparing the results of [4] and [5].

The following explains in a more intelligible way the relevance of the outcome of the above theorem (the reader might want to have [33, Prop. 12.2] in mind if seeking for a proof).

Corollary 4.14. Consider the same hypotheses of the main theorem and $0<$ $\varepsilon \leqslant N_{J_{0}}^{-n}$. Let $\varkappa$ be a positive monotonically decreasing function on $(0, \varepsilon]$ and let $0<u \leqslant \infty$. Then

$$
\left(\int_{0}^{\varepsilon}\left(\varkappa(t) \frac{f^{*}(t)}{\Phi_{q^{\prime}}(t)}\right)^{u} \mu_{q^{\prime}}(d t)\right)^{\frac{1}{u}} \leqslant c\left\|f \mid B_{p, q}^{\sigma, N}\right\|
$$


for some $c>0$ and all $f \in B_{p, q}^{\sigma, N}$ if, and only if, $\varkappa$ is bounded and $q \leqslant u \leqslant \infty$, with the modification

$$
\sup _{t \in(0, \varepsilon]} \varkappa(t) \frac{f^{*}(t)}{\Phi_{q^{\prime}}(t)} \leqslant c\left\|f \mid B_{p, q}^{\sigma, N}\right\|
$$

if $u=\infty$. Moreover, if $\varkappa$ is an arbitrary nonnegative function on $(0, \varepsilon]$, then (4.46) above holds if, and only if, $\varkappa$ is bounded.

Remark 4.15. Observe that, when $1<q \leqslant \infty$, the measure $\mu_{q^{\prime}}(d t)$ in $(4.45)$ can be replaced by

$$
\frac{d t}{\Phi_{q^{\prime}}(t)^{q^{\prime}} t^{\frac{q^{\prime}}{p}} \Lambda\left(t^{-\frac{1}{n}}\right)^{q^{\prime}} t} .
$$

Remark 4.16. Envelopes can be used to show that some conditions are necessary for something to happen. An example is in the proof of the converse to Corollary 3.10 in Corollary 4.9. Sometimes it might be necessary to take first advantage of lifts before growth envelopes can be used. An example where things work out this way can be seen in [3], in the proof of the necessity of conditions which were already known to be sufficient for the existence of an embedding between function spaces. That proof is also of interest because it takes also advantage of the knowledge of the index $q$ of the local growth envelope $\left(\Phi_{q^{\prime}}, q\right)$ in order to fulfil its task.

Acknowledgement. This research was partially supported by Unidade de Investigação Matemática e Aplicações of Universidade de Aveiro through Programa Operacional "Ciência,Tecnologia, Inovação" (POCTI) of the Fundação para a Ciência e a Tecnologia (FCT), cofinanced by the European Community fund FEDER. The second named author wishes to express his gratitude to the Universidade de Aveiro for hospitality during his visit in March 2003 when this research was initiated.

\section{References}

[1] Bennett, C. and Sharpley, R., Interpolation of Operators. Boston: Academic Press 1988.

[2] Bricchi, M. and Moura, S., Complements on growth envelopes of spaces with generalised smoothness in the sub-critical case. Z. Anal. Anwendungen 22 (2003)(2), $383-398$.

[3] Caetano, A. and Haroske, D., Continuity envelopes of spaces of generalised smoothness: a limiting case; embeddings and approximation numbers. J. Function Spaces Appl. 3 (2005), 33 - 71. 
[4] Caetano, A. and Moura, S., Local growth envelopes of spaces of generalized smoothness: the sub-critical case. Math. Nachr. 273 (2004), 43 - 57.

[5] Caetano, A. and Moura, S., Local growth envelopes of spaces of generalized smoothness: the critical case. Math. Ineq. Appl. 7 (2004), $573-606$.

[6] Edmunds, D. E. and Triebel, H., Function Spaces, Entropy Numbers, Differential Operators. Cambridge: C. U. P. 1996.

[7] Farkas, W. and H.-G. Leopold, H.-G., Characterisations of function spaces of generalised smoothness. Annali di Mat. Pura Appl. 185 (2006)(1), 1-62.

[8] Frazier, M. and Jawerth, B., Decomposition of Besov spaces. Indiana Univ. Math. J. 34 (1985), $777-799$.

[9] Frazier, M. and Jawerth, B., A discrete transform and decomposition of distribution spaces. J. Funct. Anal. 93 (1990), $34-170$.

[10] Goldman, M. L., A description of the trace space for functions of a generalized Hölder class. Dokl. Akad. Nauk SSSR 231 (1976), 525 - 528.

[11] Goldman, M. L., A description of the traces of some function spaces. Trudy Mat. Inst. Steklov 150 (1979), 99 - 127. English transl.: Proc. Steklov Inst. Math. 1981, No. 4 (150).

[12] Goldman, M. L., A method of coverings for describing general spaces of Besov type. Trudy Mat. Inst. Steklov 156 (1980), 47 - 81. English transl.: Proc. Steklov Inst. Math. 1983, No. 2 (156).

[13] Goldman, M. L., Embedding of Nikol'skii-Besov spaces with moduli of continuity of general type in Lorentz spaces. Dokl. Akad. Nauk SSSR 277 (1984), $20-24$.

[14] Goldman, M. L., On imbedding generalized Nikol'skii-Besov spaces in Lorentz spaces. Trudy Mat. Inst. Steklov 172 (1985), 128 - 139. English transl.: Proc. Steklov Inst. Math.1987, No. 3 (172).

[15] Goldman, M. L., Traces of functions with restrictions on the spectrum. Trudy Mat. Inst. Steklov 187 (1989), 69 - 77. English transl.: Proc. Steklov Inst. Math. 1990, No. 3 (187).

[16] Goldman, M. L., A criterion for the embedding of different metrics for isotropic Besov spaces with arbitrary moduli of continuity. Trudy Mat. Inst. Steklov 201 (1992), 186 - 218. English transl.: Proc. Steklov Inst. Math. 1994, No. 2 (201).

[17] Gurka, P. and Opic, B., Sharp embeddings of Besov spaces with logarithmic smoothness. Rev. Mat. Complut. 18 (2005)(1), 81 - 110.

[18] Hardy, G., Littlewood, J. E. and Pólya, G., Inequalities (reprinted second edition ed.). Cambridge: C. U. P. 1994.

[19] Haroske, D., Envelopes in function spaces - a first approach. Univ. Jena (Germany): preprint Math/Inf/16/01 2001.

[20] Kalyabin, G. A., Imbedding theorems for generalized Besov and Liouville spaces. Dokl. Akad. Nauk SSSR 232 (1977), 1245 - 1248. English transl.: Soviet Math. Dokl. 1977, no 1 (18). 
[21] Kalyabin, G. A., Trace spaces for generalized anisotropic Liouville classes. Izv. Akad. Nauk SSSR Ser. Mat. 42 (1978), 305 - 314. English transl.: Math. USSR Izv. 12 (1978).

[22] Kalyabin, G. A., A description of traces for anisotropic spaces of in classes of Triebel-Lizorkin type. Trudy Mat. Inst. Steklov 150 (1979), 160 - 173. English transl.: Proc. Steklov Inst. Math. 1981, No. 4 (150).

[23] Kalyabin, G. A., Description of functions in classes of Besov-Lizorkin-Triebel type. Trudy Mat. Inst. Steklov 156 (1980), 82 - 109. English transl.: Proc. Steklov Inst. Math. 1983, No. 2 (156).

[24] Kalyabin, G. A., Criteria for multiplicativity and imbedding in $C$ for spaces of Besov-Lizorkin-Triebel type. Mat. Zametki 30 (1981), 517 - 526. English transl.: Math. Notes 1983, No. 30.

[25] Kalyabin, G. A., Characterization of spaces of Besov-Lizorkin-Triebel type by means of generalized differences. Trudy Mat. Inst. Steklov 181 (1988), 95 - 116. English transl.: Proc. Steklov Inst. Math. 1989, No. 4 (181).

[26] Moura, S., Function spaces of generalised smoothness, entropy numbers, applications. University of Coimbra (Portugal): Ph.D. thesis 2001.

[27] Opic, B. and Trebels, W., Sharp embeddings of Bessel potential spaces with logarithmic smoothness. Math. Proc. Camb. Phil. Soc 134 (2003), 347 - 384.

[28] Runst, T. and Sickel, W., Sobolev Spaces of Fractional Order, Nemytskii Operators, and Nonlinear Partial Differential Operators. Berlin: DeGruyter 1996.

[29] Sickel, W. and Triebel, H., Hölder inequalities and sharp embeddings in function spaces of $B_{p q}^{s}$ and $F_{p q}^{s}$ type. Z. Anal. Anwendungen 14 (1995)(1), 105-140.

[30] Triebel, H., Interpolation Theory, Function Spaces, Differential Operators. Amsterdam: North Holland 1978.

[31] Triebel, H., Theory of Function Spaces. Leipzig: Geest \& Portig 1983.

[32] Triebel, H., Theory of Function Spaces II. Basel: Birkhäuser 1992.

[33] Triebel, H., The Structure of Functions. Basel: Birkhäuser 2001.

Received 13. 8. 2004 